\title{
PROOFS OF CONJECTURES OF SANDON AND ZANELLO ON COLORED PARTITION IDENTITIES
}

\author{
Bruce C. Berndt and Roberta R. Zhou
}

\begin{abstract}
In a recent systematic study, C. Sandon and F. Zanello offered 30 conjectured identities for partitions. As a consequence of their study of partition identities arising from Ramanujan's formulas for multipliers in the theory of modular equations, the present authors in an earlier paper proved three of these conjectures. In this paper, we provide proofs for the remaining 27 conjectures of Sandon and Zanello. Most of our proofs depend upon known modular equations and formulas of Ramanujan for theta functions, while for the remainder of our proofs it was necessary to derive new modular equations and to employ the process of duplication to extend Ramanujan's catalogue of theta function formulas.
\end{abstract}

\section{Introduction}

Early in this century, H. M. Farkas and I. Kra [12] began a fruitful study of partition identities arising from theta function identities and modular equations with the following elegant theorem about colored partitions.

Theorem 1.1. Let $S$ denote the set consisting of one copy of the positive integers and one additional copy of those positive integers that are multiples of 7. Then for each positive integer $k$, the number of partitions of $2 k$ into even elements of $S$ is equal to the number of partitions of $2 k+1$ into odd elements of $S$.

Shortly thereafter, it was realized that many of Ramanujan's modular equations yielded further interesting partition identities for colored partitions. For example, see papers by the first author [9], N. D. Baruah and the first author $[4],[5]$, and a paper by the present authors [10]. It is natural to ask for combinatorial proofs of these identities, and readers should consult the papers by S. O. Warnaar [17] and S. Kim [13] for beautiful arguments giving combinatorial approaches to classes of these partition identities. The work of Warnaar

Received October 14, 2013.

2010 Mathematics Subject Classification. Primary 11P84; Secondary 05A15, 05A17.

Key words and phrases. colored partitions, modular equations, theta function identities.

The first author's research was partially supported by NSA grant H98230-11-1-0200.

The second author's research was partially supported by the program of China Scholarships Council (No. 201206060076). 
and Kim motivated further proofs in the combinatorial direction by Sandon and Zanello [15]. Then in a subsequent paper [16], Sandon and Zanello offered 30 conjectures about colored partitions. As indicated in our abstract, we proved three of their conjectures in our paper [10]. Baruah and B. Boruah [6] have also established the conjectures of Sandon and Zanello.

In this paper, we establish the remaining 27 conjectures of Sandon and Zanello. We have divided our proofs into three sections. For the first nine of our proofs, we rely on known modular equations; these proofs are in Section 3. For the next six proofs, we need to develop new modular equations. In particular, we use certain "evaluations" of theta functions outside Ramanujan's catalogue of evaluations in [7, pp. 122-124]. These new formulas for theta functions are derived with the help of the classical process of duplication, which can be found in Ramanujan's notebooks [14], [7, pp. 125-126]. It is remarkable that "nice" identities exist when we go outside Ramanujan's catalogue of theta functions; usually, venturing outside the catalogue produces inelegant identities. Proofs of six identities relying on new modular equations and new formulas for theta functions are given in Section 4. Finally, in Section 5, we construct new modular equations of degree 3 to prove 12 further conjectures of Sandon and Zanello.

\section{Preliminary results}

For any complex numbers $a$ and $|q|<1$, define

$$
(a ; q)_{\infty}:=\prod_{n=0}^{\infty}\left(1-a q^{n}\right) .
$$

Recall that Ramanujan's theta functions $\varphi(-q)$ and $f(-q)$, and his function $\chi(q)$ are defined by

$$
\begin{aligned}
\varphi(-q) & :=\sum_{n=-\infty}^{\infty}(-1)^{n} q^{n^{2}}=\frac{(q ; q)_{\infty}}{(-q ; q)_{\infty}} \\
f(-q) & :=(q ; q)_{\infty} \\
\chi(q) & :=\left(-q ; q^{2}\right)_{\infty} .
\end{aligned}
$$

The latter equality in (2.1) is a consequence of Jacobi's triple product identity.

The complete elliptic integral of the first kind is defined for $|k|<1$ by

$$
K:=K(k):=\int_{0}^{\pi / 2} \frac{d \phi}{\sqrt{1-k^{2} \sin ^{2} \phi}} .
$$

The number $k$ is called the modulus. The complementary modulus $k^{\prime}$ is defined by $k^{\prime}=\sqrt{1-k^{2}}$. Set $K^{\prime}=K\left(k^{\prime}\right)$. Expanding the integrand in a binomial series and integrating termwise, we find that

$$
K=\frac{\pi}{2}{ }_{2} F_{1}\left(\frac{1}{2}, \frac{1}{2} ; 1 ; k^{2}\right)
$$


where ${ }_{2} F_{1}\left(\frac{1}{2}, \frac{1}{2} ; 1 ; k^{2}\right)$ denotes the ordinary hypergeometric function. Let $K$, $K^{\prime}, L$, and $L^{\prime}$, denote the complete elliptic integrals of the first kind associated with the moduli $k, k^{\prime}, \ell$, and $\ell^{\prime}:=\sqrt{1-\ell^{2}}$, respectively. Suppose that the equality

$$
n \frac{K^{\prime}}{K}=\frac{L^{\prime}}{L}
$$

holds for some positive integer $n$. A relation between $k$ and $\ell$ induced by (2.4) is called a modular equation of degree $n$. Ramanujan recorded his modular equations in terms of $\alpha$ and $\beta$, where $\alpha=k^{2}$ and $\beta=\ell^{2}$. We often say that $\beta$ has degree $n$ over $\alpha$.

If

$$
q:=\exp \left(-\pi \frac{{ }_{2} F_{1}\left(\frac{1}{2}, \frac{1}{2} ; 1 ; 1-\alpha\right)}{{ }_{2} F_{1}\left(\frac{1}{2}, \frac{1}{2} ; 1 ; \alpha\right)}\right)=\exp \left(-\pi \frac{K^{\prime}}{K}\right),
$$

then one of the primary theorems in the theory of elliptic functions [7, p. 101, Entry 6] asserts that

$$
\varphi^{2}(q)={ }_{2} F_{1}\left(\frac{1}{2}, \frac{1}{2} ; 1 ; \alpha\right)=: z
$$

where $\varphi(q)$ is defined by (2.1). If we further set $z_{n}:=\varphi^{2}\left(q^{n}\right)$, then the multiplier $m$ of degree $n$ is defined by

$$
m:=\frac{z_{1}}{z_{n}} .
$$

We need certain evaluations of Ramanujan for theta functions given in the following lemma [8, p. 123], [7, p. 124, Entry 12].

Lemma 2.1. If $\alpha, q$, and $z$ are related by (2.5) and (2.6), then

$$
\begin{aligned}
f(-q) & =2^{-1 / 6} \sqrt{z}(1-\alpha)^{1 / 6}(\alpha / q)^{1 / 24}, \\
f\left(-q^{2}\right) & =2^{-1 / 3} \sqrt{z}\{\alpha(1-\alpha) / q\}^{1 / 12}, \\
\chi(q) & =2^{1 / 6}\{\alpha(1-\alpha) / q\}^{-1 / 24} \\
\chi(-q) & =2^{1 / 6}(1-\alpha)^{1 / 12}(\alpha / q)^{-1 / 24}, \\
\chi\left(-q^{2}\right) & =2^{1 / 3}(1-\alpha)^{1 / 24}(\alpha / q)^{-1 / 12} .
\end{aligned}
$$

Suppose that $\beta$ has degree $n$ over $\alpha$. If we replace $q$ by $q^{n}$ above, then the same evaluations hold with $\alpha$ replaced by $\beta$ and with $z=z_{1}$ replaced by $z_{n}$.

In the following proofs, we also make use of Euler's famous identity (see [1, $3,11])$

$$
\frac{1}{\left(q ; q^{2}\right)_{\infty}}=(-q ; q)_{\infty}
$$

i.e., the number of partitions of the positive integer $n$ into odd parts is identical to the number of partitions of $n$ into distinct parts. 


\section{Proofs of nine conjectures using known modular equations}

Theorem 3.1. Let $S$ denote the set of partitions into 6 distinct colors, with the orange, blue and red parts appearing at most once, and the parts in the remaining three colors appearing at most once and only in multiples of 7 . Let $A(N)$ be the number of partitions of $2 N-2$ into even parts in $S$. Let $B(N)$ be the number of partitions of $2 N+1$ into odd parts in $S$. Then, for $N \geq 1$,

$$
4 A(N)=B(N) .
$$

Proof. Recall the modular equation for degree 7 given by [7, p. 314, Entry 19 (i)]

$$
(\alpha \beta)^{1 / 8}+\{(1-\alpha)(1-\beta)\}^{1 / 8}=1,
$$

where $m$ is the multiplier of degree 7 defined by (2.7). Taking the third power of this identity, we deduce that

$$
(\alpha \beta)^{3 / 8}+3\{\alpha(1-\alpha) \beta(1-\beta)\}^{1 / 8}+\{(1-\alpha)(1-\beta)\}^{3 / 8}=1 .
$$

Multiplying both sides of the identity (3.2) by $2 q\{\alpha(1-\alpha) \beta(1-\beta)\}^{-1 / 8}$, we find that

$$
\begin{aligned}
& \frac{8 q^{3}}{2(1-\alpha)^{1 / 8}(\alpha / q)^{-1 / 4} 2(1-\beta)^{1 / 8}\left(\beta / q^{7}\right)^{-1 / 4}} \\
& +6 q+2^{1 / 2}(1-\alpha)^{1 / 4}(\alpha / q)^{-1 / 8} 2^{1 / 2}(1-\beta)^{1 / 4}\left(\beta / q^{7}\right)^{-1 / 8} \\
= & 2^{1 / 2}\{\alpha(1-\alpha) / q\}^{-1 / 8} 2^{1 / 2}\left\{\beta(1-\beta) / q^{7}\right\}^{-1 / 8},
\end{aligned}
$$

which can be transformed into

$$
\frac{8 q^{3}}{\chi^{3}\left(-q^{2}\right) \chi^{3}\left(-q^{14}\right)}+6 q+\chi^{3}(-q) \chi^{3}\left(-q^{7}\right)=\chi^{3}(q) \chi^{3}\left(q^{7}\right)
$$

by (2.10), (2.11), and (2.12) in Lemma 2.1. Applying the definition of $\chi$ in (2.3) and Euler's identity (2.13), we obtain

$8 q^{3}\left(-q^{2} ; q^{2}\right)_{\infty}^{3}\left(-q^{14} ; q^{14}\right)_{\infty}^{3}+6 q=\left(-q ; q^{2}\right)_{\infty}^{3}\left(-q^{7} ; q^{14}\right)_{\infty}^{3}-\left(q ; q^{2}\right)_{\infty}^{3}\left(q^{7} ; q^{14}\right)_{\infty}^{3}$

Extracting the coefficients of $q^{2 N+1}$ on both sides of the identity, we complete the proof.

Remark. Theorem 3.1 is equivalent to Conjecture 3.26 in Sandon and Zanello's paper [16].

Example 3.2. Let $N=3$ in Theorem 3.1. Then $4 A(3)=B(3)=6+3\left(\begin{array}{l}3 \\ 2\end{array}\right)+$ $3\left(\begin{array}{l}3 \\ 2\end{array}\right)=24$. The relevant partitions are given by

$$
\begin{aligned}
& 4_{o}=4_{b}=4_{r}=2_{o}+2_{b}=2_{o}+2_{r}=2_{b}+2_{r} \\
& 7_{o}=7_{b}=7_{r}=7_{g}=7_{y}=7_{p}=5_{o}+1_{o}+1_{b}=\cdots=3_{o}+3_{b}+1_{o}=\cdots .
\end{aligned}
$$


Theorem 3.3. Let $S$ denote the set of partitions into 3 distinct colors, with the orange, blue, and red parts appearing at most once, and without parts in multiples of 9. Let $A(N)$ be the number of partitions of $2 N+2$ into an odd number of even parts in $S$. Let $B(N)$ be the number of partitions of $2 N-1$ into odd parts in $S$. Then, for $N \geq 1$,

$$
A(N)=B(N) .
$$

Proof. Recall the modular equation for degree 9 and its reciprocal given by [2, p. 391, Entry 17.3.24]

$$
\begin{aligned}
& \sqrt{m}=\left(\frac{\beta}{\alpha}\right)^{1 / 8}+\left(\frac{1-\beta}{1-\alpha}\right)^{1 / 8}-\left\{\frac{\beta(1-\beta)}{\alpha(1-\alpha)}\right\}^{1 / 8}, \\
& \frac{3}{\sqrt{m}}=\left(\frac{\alpha}{\beta}\right)^{1 / 8}+\left(\frac{1-\alpha}{1-\beta}\right)^{1 / 8}-\left\{\frac{\alpha(1-\alpha)}{\beta(1-\beta)}\right\}^{1 / 8},
\end{aligned}
$$

where $m$ is defined by (2.7) and $n=9$. First, taking the third power of the identity (3.3), we find that

$$
\begin{aligned}
\sqrt{m^{3}}= & \left(\frac{\beta}{\alpha}\right)^{3 / 8}+\left(\frac{1-\beta}{1-\alpha}\right)^{3 / 8}-\left\{\frac{\beta(1-\beta)}{\alpha(1-\alpha)}\right\}^{3 / 8} \\
& +3\left\{\frac{\beta(1-\beta)}{\alpha(1-\alpha)}\right\}^{1 / 8}\left\{\sqrt{m}+\left\{\frac{\beta(1-\beta)}{\alpha(1-\alpha)}\right\}^{1 / 8}\right\}(1-\sqrt{m}) \\
= & \left(\frac{\beta}{\alpha}\right)^{3 / 8}+\left(\frac{1-\beta}{1-\alpha}\right)^{3 / 8}-\left\{\frac{\beta(1-\beta)}{\alpha(1-\alpha)}\right\}^{3 / 8} \\
& +3\left\{\frac{\beta(1-\beta)}{\alpha(1-\alpha)}\right\}^{1 / 8}\left\{\sqrt{m}\left\{1-\left(\frac{\beta}{\alpha}\right)^{1 / 8}-\left(\frac{1-\beta}{1-\alpha}\right)^{1 / 8}\right\}+\left\{\frac{\beta(1-\beta)}{\alpha(1-\alpha)}\right\}^{1 / 8}\right\} .
\end{aligned}
$$

Applying the identity (3.4), we arrive at

$$
\sqrt{m^{3}}=\left(\frac{\beta}{\alpha}\right)^{3 / 8}+\left(\frac{1-\beta}{1-\alpha}\right)^{3 / 8}-\left\{\frac{\beta(1-\beta)}{\alpha(1-\alpha)}\right\}^{3 / 8}-6\left\{\frac{\beta(1-\beta)}{\alpha(1-\alpha)}\right\}^{1 / 4}
$$

Multiplying both sides of the identity (3.5) by $q^{2}\left\{\frac{\alpha(1-\alpha)}{\beta(1-\beta)}\right\}^{1 / 4}$, we have

$$
\begin{aligned}
& \frac{2^{-1} \sqrt{z_{1}^{3}}\{\alpha(1-\alpha) / q\}^{1 / 4}}{2^{-1} \sqrt{z_{9}^{3}}\left\{\beta(1-\beta) / q^{9}\right\}^{1 / 4}} \\
= & q^{3} \frac{2^{1 / 2}(1-\alpha)^{1 / 4}(\alpha / q)^{-1 / 8}}{2^{1 / 2}(1-\beta)^{1 / 4}\left(\beta / q^{9}\right)^{-1 / 8}}+\frac{2(1-\beta)^{1 / 8}\left(\beta / q^{9}\right)^{-1 / 4}}{2(1-\alpha)^{1 / 8}(\alpha / q)^{-1 / 4}} \\
& -q^{3} \frac{2^{1 / 2}\{\alpha(1-\alpha) / q\}^{-1 / 8}}{2^{1 / 2}\left\{\beta(1-\beta) / q^{9}\right\}^{-1 / 8}}-6 q^{2},
\end{aligned}
$$

which is equivalent to

$$
\frac{f^{3}\left(-q^{2}\right)}{f^{3}\left(-q^{18}\right)}=q^{3} \frac{\chi^{3}(-q)}{\chi^{3}\left(-q^{9}\right)}+\frac{\chi^{3}\left(-q^{18}\right)}{\chi^{3}\left(-q^{2}\right)}-q^{3} \frac{\chi^{3}(q)}{\chi^{3}\left(q^{9}\right)}-6 q^{2},
$$


by (2.9)-(2.12) in Lemma 2.1. Using the definitions of $f$ and $\chi$ in (2.2) and (2.3), respectively, and Euler's identity (2.13), we deduce that

$$
\frac{\left(-q^{2} ; q^{2}\right)_{\infty}^{3}}{\left(-q^{18} ; q^{18}\right)_{\infty}^{3}}-\frac{\left(q^{2} ; q^{2}\right)_{\infty}^{3}}{\left(q^{18} ; q^{18}\right)_{\infty}^{3}}=q^{3}\left\{\frac{\left(-q ; q^{2}\right)_{\infty}^{3}}{\left(-q^{9} ; q^{18}\right)_{\infty}^{3}}-\frac{\left(q ; q^{2}\right)_{\infty}^{3}}{\left(q^{9} ; q^{18}\right)_{\infty}^{3}}\right\}+6 q^{2}
$$

Equating the coefficients of $q^{2 N+2}$ on both sides of the equation, we finish the proof.

Remark. Theorem 3.3 is equivalent to Conjecture 3.28 in Sandon and Zanello's paper [16].

Example 3.4. Set $N=5$ in Theorem 3.3. Then $A(5)=B(5)=3+3\left(\begin{array}{l}3 \\ 2\end{array}\right)+$ $3^{3}+1=40$, and the derived partitions are

$$
\begin{aligned}
& 12_{o}=12_{b}=12_{r}=8_{o}+2_{o}+2_{b}=\cdots=6_{o}+4_{o}+2_{o}=\cdots=4_{o}+4_{b}+4_{r} \\
& 7_{o}+1_{o}+1_{b}=\cdots=5_{o}+3_{o}+1_{o}=\cdots=3_{o}+3_{b}+3_{r}=3_{o}+3_{b}+1_{o}+1_{b}+1_{r}=\cdots .
\end{aligned}
$$

Theorem 3.5. Let $S(T)$ denote the set of partitions into two distinct colors, with the red and blue parts appearing at most once, and without parts in multiples of 9, and with the red parts being only even (odd). Let $D_{S}(N)\left(D_{T}(N)\right)$ be the number of partitions of $N$ into an odd number of distinct elements of $S$ $(T)$. Then, for all $N \geq 2$,

$$
D_{S}(N)=D_{T}(N-1)
$$

Proof. For brevity, let $a=\left(\frac{\beta}{\alpha}\right)^{1 / 8}$ and $b=\left(\frac{1-\beta}{1-\alpha}\right)^{1 / 8}$. Multiply the identities (3.3) and (3.4) and simplify to find that

$$
0=a^{2}+b^{2}-a-b-(a+b) a b, \quad \text { or } \quad b^{2}-\sqrt{m}+a \sqrt{m}=a b^{2}+2 a b,
$$

by (3.3). Multiply both sides of this last equation by $b$ and then subtract $a \sqrt{m}$ from both sides. Thus, we see that

$$
b^{3}-(a+b-a b) \sqrt{m}=b^{3} a-a \sqrt{m}+2 a b^{2} .
$$

Applying (3.3) and dividing both sides of the last identity by $a b^{2}$, we obtain the equation

$$
\left(\frac{\alpha}{\beta}\right)^{1 / 8}\left(\frac{1-\beta}{1-\alpha}\right)^{1 / 8}-m\left(\frac{\alpha}{\beta}\right)^{1 / 8}\left(\frac{1-\alpha}{1-\beta}\right)^{1 / 4}=\left(\frac{1-\beta}{1-\alpha}\right)^{1 / 8}-\sqrt{m}\left(\frac{1-\alpha}{1-\beta}\right)^{1 / 4}+2 .
$$

Multiply both sides of the identity (3.6) by $q$ to deduce that

$$
\begin{aligned}
& \frac{2^{1 / 6}(1-\beta)^{1 / 12}\left(\beta / q^{9}\right)^{-1 / 24}}{2^{1 / 6}(1-\alpha)^{1 / 12}(\alpha / q)^{-1 / 24}} \frac{2^{1 / 3}(1-\beta)^{1 / 24}\left(\beta / q^{9}\right)^{-1 / 12}}{2^{1 / 3}(1-\alpha)^{1 / 24}(\alpha / q)^{-1 / 12}} \\
& -\frac{2^{-1 / 6} \sqrt{z_{1}}(1-\alpha)^{1 / 6}(\alpha / q)^{1 / 24}}{2^{-1 / 6} \sqrt{z_{9}}(1-\beta)^{1 / 6}\left(\beta / q^{9}\right)^{1 / 24}} \frac{2^{-1 / 3} \sqrt{z_{1}}\{\alpha(1-\alpha) / q\}^{1 / 12}}{2^{-1 / 3} \sqrt{z_{9}}\left\{\beta(1-\beta) / q^{9}\right\}^{1 / 12}} \\
= & q \frac{2^{1 / 6}(1-\beta)^{1 / 12}\left(\beta / q^{9}\right)^{-1 / 24}}{2^{1 / 6}(1-\alpha)^{1 / 12}(\alpha / q)^{-1 / 24}} \frac{2^{1 / 6}\{\alpha(1-\alpha) / q\}^{-1 / 24}}{2^{1 / 6}\left\{\beta(1-\beta) / q^{9}\right\}^{-1 / 24}}
\end{aligned}
$$




$$
-q \frac{2^{-1 / 6} \sqrt{z_{1}}(1-\alpha)^{1 / 6}(\alpha / q)^{1 / 24}}{2^{-1 / 6} \sqrt{z_{9}}(1-\beta)^{1 / 6}\left(\beta / q^{9}\right)^{1 / 24}} \frac{2^{1 / 6}(1-\alpha)^{1 / 12}(\alpha / q)^{-1 / 24}}{2^{1 / 6}(1-\beta)^{1 / 12}\left(\beta / q^{9}\right)^{-1 / 24}}+2 q,
$$

which is equivalent to

$$
\frac{\chi\left(-q^{9}\right)}{\chi(-q)} \frac{\chi\left(-q^{18}\right)}{\chi\left(-q^{2}\right)}-\frac{f(-q)}{f\left(-q^{9}\right)} \frac{f\left(-q^{2}\right)}{f\left(-q^{18}\right)}=q \frac{\chi\left(-q^{9}\right)}{\chi(-q)} \frac{\chi(q)}{\chi\left(q^{9}\right)}-q \frac{f(-q)}{f\left(-q^{9}\right)} \frac{\chi(-q)}{\chi\left(-q^{9}\right)}+2 q,
$$

by (2.8)-(2.12) in Lemma 2.1. We now employ the definitions of $f$ in $(2.2)$ and $\chi$ in (2.3), and use Euler's identity (2.13) to deduce that

$$
\begin{aligned}
& \frac{(-q ; q)_{\infty}\left(-q^{2} ; q^{2}\right)_{\infty}}{\left(-q^{9} ; q^{9}\right)_{\infty}\left(-q^{18} ; q^{18}\right)_{\infty}}-\frac{(q ; q)_{\infty}\left(q^{2} ; q^{2}\right)_{\infty}}{\left(q^{9} ; q^{9}\right)_{\infty}\left(q^{18} ; q^{18}\right)_{\infty}} \\
= & q\left\{\frac{(-q ; q)_{\infty}\left(-q ; q^{2}\right)_{\infty}}{\left(-q^{9} ; q^{9}\right)_{\infty}\left(-q^{9} ; q^{18}\right)_{\infty}}-\frac{(q ; q)_{\infty}\left(q ; q^{2}\right)_{\infty}}{\left(q^{9} ; q^{9}\right)_{\infty}\left(q^{9} ; q^{18}\right)_{\infty}}\right\}+2 q .
\end{aligned}
$$

Equating the coefficients of $q^{N}$ on both sides of the last equation, we can complete the proof.

Remark. Theorem 3.5 is equivalent to Conjecture 3.30 in Sandon and Zanello's paper [16].

Example 3.6. Set $N=9$ in Theorem 3.5. Then $D_{S}(9)=D_{T}(8)=2^{2}+1+$ $1+1+2^{2}=11$. The corresponding partitions are

$$
\begin{aligned}
& 6_{r}+2_{b}+1_{b}=\cdots=5_{b}+3_{b}+1_{b}=5_{b}+2_{b}+2_{r}=4_{r}+4_{b}+1_{b}=4_{b}+3_{b}+2_{r}=\cdots ; \\
& 8_{b}=6_{b}+1_{r}+1_{b}=5_{r}+2_{b}+1_{r}=\cdots=4_{b}+3_{b}+1_{r}=\cdots=3_{r}+3_{b}+2_{b} .
\end{aligned}
$$

Theorem 3.7. Let $S$ denote the set of partitions into six distinct colors, with the red, blue, green, and pink parts appearing at most once if they are odd or congruent to 4 modulo 8 if they are even, and the remaining two colors, orange and yellow, appearing at most once with their parts congruent to 2 modulo 4. Let $T$ denote the set of partitions into six distinct colors, with the red, blue, green, and pink parts appearing at most once with odd parts or in multiples of 8 , and the remaining two colors, orange and yellow, appearing at most once with parts congruent to 2 modulo 4 . Let $D_{S}(N)$ be the number of partitions of $N$ into an odd number of distinct elements of $S$. Let $D_{T}(N)$ denote the number of partitions of $N$ into distinct elements of $T$. Then, for all $N \geq 2$,

$$
D_{S}(N)=2 D_{T}(N-2) \text {. }
$$

Proof. Consider the modular equations of degree 4 [2, p. 386, Entry 17.3.8 (c), (d)]

$$
\begin{aligned}
m(1-\alpha)^{1 / 4}+\beta^{1 / 2} & =1, \\
\frac{4}{m} \beta^{1 / 4}+(1-\alpha)^{1 / 2} & =1,
\end{aligned}
$$

where $m$ is the multiplier of degree 4 defined by (2.7). Divide both sides of the identity (3.7) by $m(1-\alpha)^{3 / 4}$, and subtract 1 from both sides of the resulting 
identity to see that

$$
(1-\alpha)^{-1 / 2}-1+\frac{\beta^{1 / 2}}{m(1-\alpha)^{3 / 4}}=\frac{1}{m(1-\alpha)^{3 / 4}}-1 .
$$

Using (3.8) in (3.9) and then multiplying the resulting equation by

$$
m \beta^{-1 / 4}(1-\alpha)^{1 / 2}
$$

we find that

$$
4+\beta^{1 / 4}(1-\alpha)^{-1 / 4}=(1-\alpha)^{-1 / 4} \beta^{-1 / 4}-m(1-\alpha)^{1 / 2} \beta^{-1 / 4} .
$$

Multiplying both sides of $(3.10)$ by $2 q$, we rewrite (3.10) in the form

$$
\begin{aligned}
& 8 q+4 q^{2} \frac{2^{1 / 3}\{\alpha(1-\alpha) / q\}^{-1 / 12}}{2^{1 / 3}(1-\alpha)^{1 / 6}(\alpha / q)^{-1 / 12} 2^{1 / 3}\left\{\beta(1-\beta) / q^{4}\right\}^{-1 / 12} 2^{2 / 3}(1-\beta)^{1 / 12}\left(\beta / q^{4}\right)^{-1 / 6}} \\
= & \frac{2^{1 / 3}\{\alpha(1-\alpha) / q\}^{-1 / 12} 2^{1 / 3}\left\{\beta(1-\beta) / q^{4}\right\}^{-1 / 12} 2^{2 / 3}(1-\beta)^{1 / 12}\left(\beta / q^{4}\right)^{-1 / 6}}{2^{1 / 3}(1-\alpha)^{1 / 6}(\alpha / q)^{-1 / 12}} \\
& -\frac{2^{-2 / 3} z_{1}\{\alpha(1-\alpha) / q\}^{1 / 6} 2^{2 / 3}(1-\alpha)^{1 / 3}(\alpha / q)^{-1 / 6} 2^{1 / 3}(1-\beta)^{1 / 6}\left(\beta / q^{4}\right)^{-1 / 12}}{2^{-2 / 3} z_{4}\left\{\beta(1-\beta) / q^{4}\right\}^{1 / 6}},
\end{aligned}
$$

which is equivalent to

$$
8 q+4 q^{2} \frac{\chi^{2}(q)}{\chi^{2}(-q) \chi^{2}\left(q^{4}\right) \chi^{2}\left(-q^{8}\right)}=\frac{\chi^{2}(q) \chi^{2}\left(q^{4}\right) \chi^{2}\left(-q^{8}\right)}{\chi^{2}(-q)}-\frac{f^{2}\left(-q^{2}\right) \chi^{4}(-q) \chi^{2}\left(-q^{4}\right)}{f^{2}\left(-q^{8}\right)},
$$

by (2.9)-(2.12) in Lemma 2.1. We apply (2.2), (2.3), and Euler's identity (2.13) to obtain

$$
\begin{aligned}
& 8 q+4 q^{2} \frac{(-q ; q)_{\infty}^{2}\left(-q ; q^{2}\right)_{\infty}^{2}\left(-q^{8} ; q^{8}\right)_{\infty}^{2}}{\left(-q^{4} ; q^{8}\right)_{\infty}^{2}} \\
= & \frac{(-q ; q)_{\infty}^{2}\left(-q ; q^{2}\right)_{\infty}^{2}\left(-q^{4} ; q^{8}\right)_{\infty}^{2}}{\left(-q^{8} ; q^{8}\right)_{\infty}^{2}}-\frac{(q ; q)_{\infty}^{2}\left(q ; q^{2}\right)_{\infty}^{2}\left(q^{4} ; q^{8}\right)_{\infty}^{2}}{\left(q^{8} ; q^{8}\right)_{\infty}^{2}} .
\end{aligned}
$$

Equate the coefficients of $q^{N}$ on both sides of the equation to finish the proof.

Remark. Theorem 3.7 is equivalent to Conjecture 3.31 in Sandon and Zanello's paper [16].

Example 3.8. Let $N=6$ in Theorem 3.7. Then $D_{S}(6)=2+4\left(\begin{array}{l}4 \\ 2\end{array}\right)+2 \cdot 4^{2}+2=$ 60 and $D_{T}(4)=4^{2}+1+2\left(\begin{array}{l}4 \\ 2\end{array}\right)+1=30$. The corresponding partitions are given by

$$
\begin{aligned}
& 6_{o}=6_{y}=4_{r}+1_{r}+1_{b}=\cdots=3_{r}+2_{o}+1_{r}=\cdots=2_{o}+1_{r}+1_{b}+1_{g}+1_{p}=\cdots ; \\
& 3_{r}+1_{r}=\cdots=2_{o}+2_{y}=2_{o}+1_{r}+1_{b}=\cdots=1_{r}+1_{b}+1_{g}+1_{p} .
\end{aligned}
$$

Theorem 3.9. Let $S$ denote the set of partitions into eight distinct colors, with the red, blue, and green parts appearing at most once without parts in multiples of 3 , with the pink parts appearing at most once with parts in multiples of 3 but not multiples of 9 , and with the last four colors appearing at most once with 
parts in multiples of 9 . Let $A(N)$ denote the number of partitions of $2 N+1$ into odd parts in $S$, and let $B(N)$ denote the number of partitions of $2 N-2$ into even parts in $S$. Then, for all $N \geq 1$,

$$
A(N)=2 B(N) \text {. }
$$

Proof. If $\alpha, \beta$, and $\gamma$ are of the first, third, and ninth degrees, respectively, then $[7$, p. $232,(5.1)]$

$$
\begin{aligned}
& \left(\frac{\alpha^{3}}{\beta}\right)^{1 / 8}=\frac{3+m}{2 m}, \quad\left\{\frac{(1-\alpha)^{3}}{1-\beta}\right\}^{1 / 8}=\frac{3-m}{2 m} \\
& \left(\frac{\gamma^{3}}{\beta}\right)^{1 / 8}=\frac{m^{\prime}-1}{2}, \quad\left\{\frac{(1-\gamma)^{3}}{1-\beta}\right\}^{1 / 8}=\frac{m^{\prime}+1}{2},
\end{aligned}
$$

where $m=z_{1} / z_{3}$, and $m^{\prime}=z_{3} / z_{9}$. Multiplying the corresponding identities and adding the resulting equations, we can check that

$$
3 \frac{m^{\prime}}{m}=1+2\left(\frac{\alpha^{3} \gamma^{3}}{\beta^{2}}\right)^{1 / 8}+2\left\{\frac{(1-\alpha)^{3}(1-\gamma)^{3}}{(1-\beta)^{2}}\right\}^{1 / 8} .
$$

Using the identity [7, p. 352, Entry 3 (iii)]

$$
1-2^{4 / 3}\left\{\frac{\alpha^{3} \gamma^{3}(1-\alpha)^{3}(1-\gamma)^{3}}{\beta^{2}(1-\beta)^{2}}\right\}^{1 / 24}=\frac{m^{\prime}}{m},
$$

we obtain a new modular equation

$1-\left\{\frac{(1-\alpha)^{3}(1-\gamma)^{3}}{(1-\beta)^{2}}\right\}^{1 / 8}=\left(\frac{\alpha^{3} \gamma^{3}}{\beta^{2}}\right)^{1 / 8}+3 \cdot 2^{1 / 3}\left\{\frac{\alpha^{3} \gamma^{3}(1-\alpha)^{3}(1-\gamma)^{3}}{\beta^{2}(1-\beta)^{2}}\right\}^{1 / 24}$.

Multiplying both sides of the identity (3.12) by $2^{2 / 3} q\left\{\frac{\alpha^{3} \gamma^{3}(1-\alpha)^{3}(1-\gamma)^{3}}{\beta^{2}(1-\beta)^{2}}\right\}^{-1 / 24}$, we have

$$
\begin{gathered}
\frac{\left\{2^{1 / 6}\{\alpha(1-\alpha) / q\}^{-1 / 24}\right\}^{3}\left\{2^{1 / 6}\left\{\gamma(1-\gamma) / q^{9}\right\}^{-1 / 24}\right\}^{3}}{\left\{2^{1 / 6}\left\{\beta(1-\beta) / q^{3}\right\}^{-1 / 24}\right\}^{2}} \\
-\frac{\left\{2^{1 / 6}(1-\alpha)^{1 / 12}(\alpha / q)^{-1 / 24}\right\}^{3}\left\{2^{1 / 6}(1-\gamma)^{1 / 12}\left(\gamma / q^{9}\right)^{-1 / 24}\right\}^{3}}{\left\{2^{1 / 6}(1-\beta)^{1 / 12}\left(\beta / q^{3}\right)^{-1 / 24}\right\}^{2}} \\
=4 q^{3} \frac{\left\{2^{1 / 3}(1-\beta)^{1 / 24}\left(\beta / q^{3}\right)^{-1 / 12}\right\}^{2}}{\left\{2^{1 / 3}(1-\alpha)^{1 / 24}(\alpha / q)^{-1 / 12}\right\}^{3}\left\{2^{1 / 3}(1-\gamma)^{1 / 24}\left(\gamma / q^{9}\right)^{-1 / 12}\right\}^{3}}+6 q,
\end{gathered}
$$

which is equivalent to

$$
\frac{\chi^{3}(q) \chi^{3}\left(q^{9}\right)}{\chi^{2}\left(q^{3}\right)}-\frac{\chi^{3}(-q) \chi^{3}\left(-q^{9}\right)}{\chi^{2}\left(-q^{3}\right)}=4 q^{3} \frac{\chi^{2}\left(-q^{6}\right)}{\chi^{3}\left(-q^{2}\right) \chi^{3}\left(-q^{18}\right)}+6 q,
$$

by (2.9)-(2.12) in Lemma 2.1. We finally use (2.2), (2.3), and Euler's identity $(2.13)$ to find that

$$
\frac{\left(-q ; q^{2}\right)_{\infty}^{3}\left(-q^{9} ; q^{18}\right)_{\infty}^{3}}{\left(-q^{3} ; q^{6}\right)_{\infty}^{2}}-\frac{\left(q ; q^{2}\right)_{\infty}^{3}\left(q^{9} ; q^{18}\right)_{\infty}^{3}}{\left(q^{3} ; q^{6}\right)_{\infty}^{2}}=4 q^{3} \frac{\left(-q^{2} ; q^{2}\right)_{\infty}^{3}\left(-q^{18} ; q^{18}\right)_{\infty}^{3}}{\left(-q^{6} ; q^{6}\right)_{\infty}^{2}}+6 q .
$$


Equating the coefficients of $q^{2 N+1}$ on both sides of the equation, we finish the proof.

Remark. Theorem 3.9 is equivalent to Conjecture 3.38 in Sandon and Zanello's paper [16].

Example 3.10. Set $N=4$ in Theorem 3.9. Then $A(4)=2 B(4)=4+3\left(\begin{array}{l}3 \\ 2\end{array}\right)+$ $3^{2}=22=2 \times\left(1+3^{2}+1\right)$. The relevant partitions are given by

$$
\begin{aligned}
& 9_{o}=9_{y}=\cdots=7_{r}+1_{r}+1_{b}=\cdots=5_{r}+3_{p}+1_{r}=\cdots ; \\
& 6_{p}=4_{r}+2_{r}=\cdots=2_{r}+2_{b}+2_{g} .
\end{aligned}
$$

Theorem 3.11. Let $S$ denote the set of partitions into four distinct colors, with the red and blue parts appearing at most once without multiples of 10 , the green parts appearing at most once with odd parts, and the orange parts appearing at most once in only odd multiples of 5. Let $T$ denote the set of partitions into four distinct colors, with the red and blue parts appearing at most once without odd multiples of 5 , the green parts appearing at most once with even parts, and the orange parts appearing at most once in only multiples of 10. Let $D_{S}(N)$ denote the number of partitions of $N$ into an odd number of distinct elements of $S$, and let $D_{T}(N)$ be the number of partitions of $N$ into distinct elements of $T$. Then, for all $N \geq 2$,

$$
D_{S}(N)=2 D_{T}(N-2) \text {. }
$$

Proof. Consider the modular equations for degree 5 [7, p. 280, Entry 13 (iv), (v), (vii)]

$$
\begin{aligned}
& m=1+2^{4 / 3}\left\{\frac{\beta^{5}(1-\beta)^{5}}{\alpha(1-\alpha)}\right\}^{1 / 24}, \\
& m=\frac{1+\left\{\frac{(1-\beta)^{5}}{1-\alpha}\right\}^{1 / 8}}{1+\left\{(1-\alpha)^{3}(1-\beta)\right\}^{1 / 8}}, \\
& \left(\alpha \beta^{3}\right)^{1 / 8}+\left\{(1-\alpha)(1-\beta)^{3}\right\}^{1 / 8}=1-2^{1 / 3}\left\{\frac{\beta^{5}(1-\alpha)^{5}}{\alpha(1-\beta)}\right\}^{1 / 24},
\end{aligned}
$$

where $m$ is defined by (2.7). First rewrite (3.14) in the form

$$
-m\left\{(1-\alpha)^{3}(1-\beta)\right\}^{1 / 8}=-\left\{\frac{(1-\beta)^{5}}{1-\alpha}\right\}^{1 / 8}+m-1 .
$$

Using (3.13) in (3.16), multiplying both sides of the resulting equation by (1$\alpha)^{1 / 4}(1-\beta)^{-1 / 4}$, and then adding 1 on both sides of that identity, we deduce that

(3.17) $1-m\left\{\frac{(1-\alpha)^{5}}{1-\beta}\right\}^{1 / 8}=1-\left\{(1-\alpha)(1-\beta)^{3}\right\}^{1 / 8}+2 \cdot 2^{1 / 3}\left\{\frac{\beta^{5}(1-\alpha)^{5}}{\alpha(1-\beta)}\right\}^{1 / 24}$.

Utilizing equation (3.15), we find from (3.17) that

$$
1-m\left\{\frac{(1-\alpha)^{5}}{1-\beta}\right\}^{1 / 8}=\left(\alpha \beta^{3}\right)^{1 / 8}+3 \cdot 2^{1 / 3}\left\{\frac{\beta^{5}(1-\alpha)^{5}}{\alpha(1-\beta)}\right\}^{1 / 24}
$$


Multiply both sides of the identity (3.18) by $2^{2 / 3} q\left\{\frac{\alpha(1-\beta)}{\beta^{5}(1-\alpha)^{5}}\right\}^{1 / 24}$ to deduce that

$$
\begin{aligned}
& \frac{\left\{2^{1 / 3}(1-\beta)^{1 / 24}\left(\beta / q^{5}\right)^{-1 / 12}\right\}^{2}\left\{2^{1 / 6}\{\alpha(1-\alpha) / q\}^{-1 / 24}\right\}^{3} 2^{1 / 6}\left\{\beta(1-\beta) / q^{5}\right\}^{-1 / 24}}{\left\{2^{1 / 3}(1-\alpha)^{1 / 24}(\alpha / q)^{-1 / 12}\right\}^{2}} \\
& -\frac{\left\{2^{-1 / 3} \sqrt{z_{1}}\{\alpha(1-\alpha) / q\}^{1 / 12}\right\}^{2}\left\{2^{1 / 6}(1-\alpha)^{1 / 12}(\alpha / q)^{-1 / 24}\right\}^{3} 2^{1 / 6}(1-\beta)^{1 / 12}\left(\beta / q^{5}\right)^{-1 / 24}}{\left\{2^{-1 / 3} \sqrt{z_{5}}\left\{\beta(1-\beta) / q^{5}\right\}^{1 / 12}\right\}^{2}} \\
= & \frac{4 q^{2}\left\{2^{1 / 6}\{\alpha(1-\alpha) / q\}^{-1 / 24}\right\}^{2}}{\left\{2^{1 / 3}(1-\alpha)^{1 / 24}(\alpha / q)^{-1 / 12}\right\}^{3}\left\{2^{1 / 6}\left\{\beta(1-\beta) / q^{5}\right\}^{-1 / 24}\right\}^{2} 2^{1 / 3}(1-\beta)^{1 / 24}\left(\beta / q^{5}\right)^{-1 / 12}}+6 q,
\end{aligned}
$$

which is equivalent to

$$
\frac{\chi^{2}\left(-q^{10}\right) \chi^{3}(q) \chi\left(q^{5}\right)}{\chi^{2}\left(-q^{2}\right)}-\frac{f^{2}\left(-q^{2}\right) \chi^{3}(-q) \chi\left(-q^{5}\right)}{f^{2}\left(-q^{10}\right)}=4 q^{2} \frac{\chi^{2}(q)}{\chi^{3}\left(-q^{2}\right) \chi^{2}\left(q^{5}\right) \chi\left(-q^{10}\right)}+6 q,
$$

by (2.9)-(2.12) in Lemma 2.1. Applying the definitions of $f$ in $(2.2)$ and $\chi$ in (2.3), and Euler's identity (2.13), we then obtain

$$
\begin{aligned}
& \frac{(-q ; q)_{\infty}^{2}\left(-q ; q^{2}\right)_{\infty}\left(-q^{5} ; q^{10}\right)_{\infty}}{\left(-q^{10} ; q^{10}\right)_{\infty}^{2}}-\frac{(q ; q)_{\infty}^{2}\left(q ; q^{2}\right)_{\infty}\left(q^{5} ; q^{10}\right)_{\infty}}{\left(q^{10} ; q^{10}\right)_{\infty}^{2}} \\
= & 4 q^{2} \frac{(-q ; q)_{\infty}^{2}\left(-q^{2} ; q^{2}\right)_{\infty}\left(-q^{10} ; q^{10}\right)_{\infty}}{\left(-q^{5} ; q^{10}\right)_{\infty}^{2}}+6 q .
\end{aligned}
$$

Equating the coefficients of $q^{N}$ on both sides of the equation, we complete the proof.

Remark. Theorem 3.11 is equivalent to Conjecture 3.39 in Sandon and Zanello's paper [16].

Example 3.12. Let $N=7$ in Theorem 3.11. Then $D_{S}(7)=3+4\left(\begin{array}{l}3 \\ 2\end{array}\right)+2^{2} \cdot 3+$ $3\left(\begin{array}{l}3 \\ 2\end{array}\right)+3+1=40$ and $D_{T}(5)=3 \cdot 2+2 \cdot 3+2+2\left(\begin{array}{l}3 \\ 2\end{array}\right)=20$, with the relevant partitions given by

$$
\begin{aligned}
& 7_{r}=7_{b}=7_{g}=5_{o}+1_{r}+1_{b}=\cdots=4_{r}+2_{r}+1_{g}=\cdots=3_{r}+3_{b}+1_{g}=\cdots \\
& \quad=3_{g}+2_{r}+2_{b}=\cdots=2_{r}+2_{b}+1_{r}+1_{b}+1_{g} ; \\
& 4_{g}+1_{r}=\cdots=3_{r}+2_{g}=\cdots=3_{r}+1_{r}+1_{b}=\cdots=2_{r}+2_{b}+1_{r}=\cdots .
\end{aligned}
$$

Theorem 3.13. Let $S$ denote the set of partitions into six distinct colors, with the red and blue parts appearing at most once with odd parts, the green parts appearing at most once with even parts, the orange and pink parts appearing at most once and only in odd multiples of 7 , and the yellow parts appearing at most once and only in multiples of 14 . Let $T$ denote the set of partitions into six distinct colors, with the red and blue parts appearing at most once with even parts, the green parts appearing at most once with odd parts, the orange and pink parts appearing at most once and only in multiples of 14, and the yellow parts appearing at most once and only in odd multiples of 7 . Let $D_{S}(N)$ be the number of partitions of $N$ into distinct elements of $S$. Let $D_{T}(N)$ be the number of partitions of $N$ into distinct elements of $T$. Then, for all $N \geq 1$,

$$
D_{S}(N)=2 D_{T}(N-1) \text {. }
$$


Proof. We begin with the modular equation of degree 7 given in (3.1). Divide both sides of $(3.1)$ by $(1-\alpha)^{1 / 8}(1-\beta)^{1 / 8}$ and rewrite the resulting identity as

$$
=\frac{\frac{2^{1 / 6}\{\alpha(1-\alpha) / q\}^{-1 / 24} 2^{1 / 6}\left\{\beta(1-\beta) / q^{7}\right\}^{-1 / 24}}{2^{1 / 6}(1-\alpha)^{1 / 12}(\alpha / q)^{-1 / 24} 2^{1 / 6}(1-\beta)^{1 / 12}\left(\beta / q^{7}\right)^{-1 / 24}}}{(1-\alpha)^{1 / 12}(\alpha / q)^{-1 / 24}(1-\alpha)^{1 / 24}(\alpha / q)^{-1 / 12}(1-\beta)^{1 / 12}\left(\beta / q^{7}\right)^{-1 / 24}(1-\beta)^{1 / 24}\left(\beta / q^{7}\right)^{-1 / 12}}+1,
$$

which is equivalent to

$$
\frac{\chi(q) \chi\left(q^{7}\right)}{\chi(-q) \chi\left(-q^{7}\right)}=\frac{2 q}{\chi(-q) \chi\left(-q^{2}\right) \chi\left(-q^{7}\right) \chi\left(-q^{14}\right)}+1,
$$

by (2.10)-(2.12) in Lemma 2.1. Employing (2.3), and invoking Euler's identity (2.13), we then can check that

$$
\begin{aligned}
& (-q ; q)_{\infty}\left(-q ; q^{2}\right)_{\infty}\left(-q^{7} ; q^{7}\right)_{\infty}\left(-q^{7} ; q^{14}\right)_{\infty} \\
= & 2 q(-q ; q)_{\infty}\left(-q^{2} ; q^{2}\right)_{\infty}\left(-q^{7} ; q^{7}\right)_{\infty}\left(-q^{14} ; q^{14}\right)_{\infty}+1 .
\end{aligned}
$$

Equate the coefficients of $q^{N}$ on both sides of the equation to complete the proof.

Remark. Theorem 3.13 is equivalent to Conjecture 3.51 in Sandon and Zanello's paper [16].

Example 3.14. Set $N=7$ in Theorem 3.13. Then $D_{S}(7)=18$ and $D_{T}(6)=$ $2+1+2^{2}+2=9$, and the relevant partitions are

$$
\begin{aligned}
7_{r} & =7_{b}=7_{o}=7_{p}=6_{g}+1_{r}=6_{b}+1_{r}=5_{r}+2_{g}=5_{b}+2_{g}=5_{r}+1_{r}+1_{b}=5_{b}+1_{r}+1_{b} \\
& =4_{g}+3_{r}=4_{g}+3_{b}=4_{g}+2_{g}+1_{r}=4_{g}+2_{g}+1_{b}=3_{r}+3_{b}+1_{r}=\cdots \\
& =3_{r}+2_{g}+1_{r}+1_{b}=\cdots ; \\
6_{r} & =6_{b}=5_{g}+1_{g}=4_{r}+2_{b}=\cdots=3_{g}+2_{r}+1_{g}=3_{g}+2_{b}+1_{g} .
\end{aligned}
$$

Theorem 3.15. Let $S$ denote the set of partitions into four distinct colors, with the red and blue parts appearing at most once with odd parts, the green parts appearing at most once with even parts but not multiples of 16, and the orange parts appearing at most once and only with odd multiples of 8 . Let $T$ denote the set of partitions into four distinct colors, with the red and blue parts appearing at most once with odd parts, the green parts appearing at most once with even parts but not odd multiples of 8, and the orange parts appearing at most once in only multiples of 16 . Let $D_{S}(N)$ be the number of partitions of $N$ into an odd number of distinct elements of $S$. Let $D_{T}(N)$ be the number of partitions of $N$ into distinct elements of $T$. Then, for all $N \geq 2$,

$$
D_{S}(N)=D_{T}(N-2) \text {. }
$$

Proof. Recall the modular equations for degree 8 [2, p. 386, Entry 17.3.9]

$$
\sqrt{m}(1-\alpha)^{1 / 8}+\beta^{1 / 4}=1,
$$




$$
\frac{2^{3 / 2}}{\sqrt{m}} \beta^{1 / 8}+(1-\alpha)^{1 / 4}=1
$$

where $m$ is defined by (2.7) and $n=8$. Divide both sides of the identity (3.19) by $\sqrt{m}(1-\alpha)^{3 / 8}$ and subtract 1 from both sides of the resulting identity. Then we can check that

$$
(1-\alpha)^{-1 / 4}-1+\frac{\beta^{1 / 4}}{\sqrt{m}(1-\alpha)^{3 / 8}}=\frac{1}{\sqrt{m}(1-\alpha)^{3 / 8}}-1 .
$$

Using equation (3.20) in (3.21) and multiplying the resulting identity by

$$
\sqrt{m} \beta^{-1 / 8}(1-\alpha)^{1 / 4}
$$

we find that

$$
2^{3 / 2}+\beta^{1 / 8}(1-\alpha)^{-1 / 8}=(1-\alpha)^{-1 / 8} \beta^{-1 / 8}-\sqrt{m}(1-\alpha)^{1 / 4} \beta^{-1 / 8} .
$$

Multiplying both sides of the identity $(3.22)$ by $2^{1 / 2} q$, we arrive at

$$
\begin{aligned}
& 4 q+2 q^{2} \frac{2^{1 / 3}\{\alpha(1-\alpha) / q\}^{-1 / 12}}{2^{1 / 3}(1-\alpha)^{1 / 24}(\alpha / q)^{-1 / 12} 2^{1 / 6}\left\{\beta(1-\beta) / q^{8}\right\}^{-1 / 24} 2^{1 / 3}(1-\beta)^{1 / 24}\left(\beta / q^{8}\right)^{-1 / 12}} \\
= & \frac{2^{1 / 3}\{\alpha(1-\alpha) / q\}^{-1 / 12} 2^{1 / 6}\left\{\beta(1-\beta) / q^{8}\right\}^{-1 / 24} 2^{1 / 3}(1-\beta)^{1 / 24}\left(\beta / q^{8}\right)^{-1 / 12}}{2^{1 / 3}(1-\alpha)^{1 / 24}(\alpha / q)^{-1 / 12}} \\
& -\frac{2^{-1 / 3} \sqrt{z_{1}}\{\alpha(1-\alpha) / q\}^{1 / 12} 2^{1 / 3}(1-\alpha)^{1 / 6}(\alpha / q)^{-1 / 12} 2^{1 / 6}(1-\beta)^{1 / 12}\left(\beta / q^{8}\right)^{-1 / 24}}{2^{-1 / 3} \sqrt{z_{8}}\left\{\beta(1-\beta) / q^{8}\right\}^{1 / 12}}
\end{aligned}
$$

which is equivalent to

$$
4 q+2 q^{2} \frac{\chi^{2}(q)}{\chi\left(-q^{2}\right) \chi\left(q^{8}\right) \chi\left(-q^{16}\right)}=\frac{\chi^{2}(q) \chi\left(q^{8}\right) \chi\left(-q^{16}\right)}{\chi\left(-q^{2}\right)}-\frac{f\left(-q^{2}\right) \chi^{2}(-q) \chi\left(-q^{8}\right)}{f\left(-q^{16}\right)},
$$

by (2.9)-(2.12) in Lemma 2.1. Employing (2.2) and (2.3), and invoking Euler's identity (2.13), we then can check that

$$
\begin{aligned}
& 4 q+2 q^{2} \frac{\left(-q ; q^{2}\right)_{\infty}^{2}\left(-q^{2} ; q^{2}\right)_{\infty}\left(-q^{16} ; q^{16}\right)_{\infty}}{\left(-q^{8} ; q^{16}\right)_{\infty}} \\
= & \frac{\left(-q ; q^{2}\right)_{\infty}^{2}\left(-q^{2} ; q^{2}\right)_{\infty}\left(-q^{8} ; q^{16}\right)_{\infty}}{\left(-q^{16} ; q^{16}\right)_{\infty}}-\frac{\left(q ; q^{2}\right)_{\infty}^{2}\left(q^{2} ; q^{2}\right)_{\infty}\left(q^{8} ; q^{16}\right)_{\infty}}{\left(q^{16} ; q^{16}\right)_{\infty}} .
\end{aligned}
$$

Equate the coefficients of $q^{N}$ on both sides of the equation to finish the proof.

Remark. Theorem 3.15 is equivalent to Conjecture 3.52 in Sandon and Zanello's paper [16].

Example 3.16. Set $N=8$ in Theorem 3.15. Then $D_{S}(8)=D_{T}(6)=4+2^{2}+$ $2^{2}=12$, and the corresponding partitions are

$$
\begin{aligned}
& 8_{o}=8_{g}=6_{g}+1_{r}+1_{b}=5_{r}+2_{g}+1_{r}=\cdots=4_{g}+3_{r}+1_{r}=\cdots=3_{r}+3_{b}+2_{g} ; \\
& 6_{o}=5_{r}+1_{b}=\cdots=4_{o}+2_{o}=4_{o}+1_{r}+1_{b}=3_{r}+3_{b}=3_{r}+2_{o}+1_{r}=\cdots .
\end{aligned}
$$


Theorem 3.17. Let $S$ denote the set of partitions into four distinct colors, with the red parts appearing at most once with odd parts, the blue parts appearing at most once with odd multiples of 3 , the green parts appearing at most once with odd multiples of 5, and the orange parts appearing at most once in only odd multiples of 15 . Let $T$ denote the set of partitions into four distinct colors, with the red parts appearing at most once with even parts, the blue parts appearing at most once with multiples of 6 , the green parts appearing at most once with multiples of 10 , and the orange parts appearing at most once in only multiples of 30. Let $D_{S}(N)$ be the number of partitions of $N$ into an odd number of distinct elements of $S$. Let $D_{T}(N)$ be the number of partitions of $N$ into distinct elements of $T$. Then, for all $N \geq 3$,

$$
D_{S}(N)=2 D_{T}(N-3) \text {. }
$$

Proof. Let $\alpha, \beta, \gamma$, and $\delta$ be of the first, third, fifth, and fifteenth degrees, respectively. Then [7, p. 385, Entry 11 (xiv)]

$$
\begin{aligned}
& (\alpha \beta \gamma \delta)^{1 / 8}+\{(1-\alpha)(1-\beta)(1-\gamma)(1-\delta)\}^{1 / 8} \\
& +2^{1 / 3}\{\alpha \beta \gamma \delta(1-\alpha)(1-\beta)(1-\gamma)(1-\delta)\}^{1 / 24}=1 .
\end{aligned}
$$

Multiply both sides of the identity (3.23) by $\{\alpha \beta \gamma \delta(1-\alpha)(1-\beta)(1-\gamma)(1-$ $\delta)\}^{-1 / 24} q$ and rearrange the resulting identity to arrive at

$$
\begin{aligned}
& \frac{q}{\{\alpha \beta \gamma \delta(1-\alpha)(1-\beta)(1-\gamma)(1-\delta)\}^{1 / 24}}-\frac{\{(1-\alpha)(1-\beta)(1-\gamma)(1-\delta)\}^{1 / 12} q}{(\alpha \beta \gamma \delta)^{1 / 24}} \\
= & \frac{(\alpha \beta \gamma \delta)^{1 / 12} q}{\{(1-\alpha)(1-\beta)(1-\gamma)(1-\delta)\}^{1 / 24}}+2^{1 / 3} q,
\end{aligned}
$$

which is equivalent to

$$
\begin{aligned}
& 2^{1 / 6}\{\alpha(1-\alpha) / q\}^{-1 / 24} 2^{1 / 6}\left\{\beta(1-\beta) / q^{3}\right\}^{-1 / 24} 2^{1 / 6}\left\{\gamma(1-\gamma) / q^{5}\right\}^{-1 / 24} 2^{1 / 6}\left\{\delta(1-\delta) / q^{15}\right\}^{-1 / 24} \\
& -2^{2 / 3}(1-\alpha)^{1 / 12}(\alpha / q)^{-1 / 24}(1-\beta)^{1 / 12}\left(\beta / q^{3}\right)^{-1 / 24}(1-\gamma)^{1 / 12}\left(\gamma / q^{5}\right)^{-1 / 24}(1-\delta)^{1 / 12}\left(\delta / q^{15}\right)^{-1 / 24} \\
= & \frac{4 q^{3}}{2^{4 / 3}(1-\alpha)^{1 / 24}(\alpha / q)^{-1 / 12}(1-\beta)^{1 / 24}\left(\beta / q^{3}\right)^{-1 / 12}(1-\gamma)^{1 / 24}\left(\gamma / q^{5}\right)^{-1 / 12}(1-\delta)^{1 / 24}\left(\delta / q^{15}\right)^{-1 / 12}}+2 q .
\end{aligned}
$$

Employing (2.10)-(2.12), we arrive at

$$
\begin{aligned}
& \chi(q) \chi\left(q^{3}\right) \chi\left(q^{5}\right) \chi\left(q^{15}\right)-\chi(-q) \chi\left(-q^{3}\right) \chi\left(-q^{5}\right) \chi\left(-q^{15}\right) \\
= & \frac{4 q^{3}}{\chi\left(-q^{2}\right) \chi\left(-q^{6}\right) \chi\left(-q^{10}\right) \chi\left(-q^{30}\right)}+2 q .
\end{aligned}
$$

Applying (2.3) and invoking Euler's identity (2.13), we then can find that

$$
\begin{aligned}
& \left(-q ; q^{2}\right)_{\infty}\left(-q^{3} ; q^{6}\right)_{\infty}\left(-q^{5} ; q^{10}\right)_{\infty}\left(-q^{15} ; q^{30}\right)_{\infty} \\
& -\left(q ; q^{2}\right)_{\infty}\left(q^{3} ; q^{6}\right)_{\infty}\left(q^{5} ; q^{10}\right)_{\infty}\left(q^{15} ; q^{30}\right)_{\infty} \\
= & 4 q^{3}\left(-q^{2} ; q^{2}\right)_{\infty}\left(-q^{6} ; q^{6}\right)_{\infty}\left(-q^{10} ; q^{10}\right)_{\infty}\left(-q^{30} ; q^{30}\right)_{\infty}+2 q .
\end{aligned}
$$


Equate the coefficients of $q^{N}$ on both sides of the equation to complete the proof.

Remark. Theorem 3.17 is equivalent to Conjecture 3.53 in Sandon and Zanello's paper [16].

Example 3.18. Set $N=9$ in Theorem 3.17. Then $D_{S}(9)=2 D_{T}(6)=6$, and the corresponding partitions are

$$
\begin{aligned}
& 9_{r}=9_{b}=5_{r}+3_{r}+1_{r}=5_{g}+3_{r}+1_{r}=5_{r}+3_{b}+1_{r}=5_{g}+3_{b}+1_{r} ; \\
& 6_{r}=6_{b}=4_{r}+2_{r} .
\end{aligned}
$$

\section{Proofs of six conjectures using new modular equations, I}

Before giving proofs of the next six theorems, we record and derive some useful formulas. First [7, p. 122, Entry 10 (ii), (iii)],

$$
\begin{aligned}
\varphi(-q) & =\sqrt{z}(1-\alpha)^{1 / 4}, \\
\varphi\left(-q^{2}\right) & =\sqrt{z}(1-\alpha)^{1 / 8},
\end{aligned}
$$

and using (4.2), we employ the process of duplication [7, p. 125] to find that

$$
\varphi\left(-q^{4}\right)=\sqrt{z} 2^{-1 / 4}(1-\alpha)^{1 / 16}(1+\sqrt{1-\alpha})^{1 / 4} .
$$

Employing the principle of duplication to (4.3), we find that

$$
\varphi\left(-q^{8}\right)=\sqrt{z} 2^{-5 / 8}(1-\alpha)^{1 / 32}(1+\sqrt{1-\alpha})^{1 / 8}\left(1+(1-\alpha)^{1 / 4}\right)^{1 / 2} .
$$

Second, recall that $[7$, p. 122 , Entry 10 (iv), (v)]

$$
\begin{gathered}
\varphi\left(q^{2}\right)=\sqrt{z}\left(\frac{1}{2}(1+\sqrt{1-\alpha})\right)^{1 / 2}, \\
\varphi\left(q^{4}\right)=\frac{1}{2} \sqrt{z}\left(1+(1-\alpha)^{1 / 4}\right) .
\end{gathered}
$$

Applying the duplication process to the last formula, we derive that

$$
\varphi\left(q^{8}\right)=\sqrt{z} 2^{-3 / 2}\left((1+\sqrt{1-\alpha})^{1 / 2}+\sqrt{2}(1-\alpha)^{1 / 8}\right) .
$$

Turning our attention now to $f\left(-q^{n}\right)$, when $n$ is a power of 2 , recall that $[7$, p. 124, Entry 12 (iv)]

$$
f\left(-q^{4}\right)=\sqrt{z} 2^{-2 / 3}(1-\alpha)^{1 / 24}(\alpha / q)^{1 / 6} .
$$

Applying the process of duplication to (4.8), we see that

$$
f\left(-q^{8}\right)=\sqrt{z} 2^{-13 / 12}(1-\alpha)^{1 / 48}(1+\sqrt{1-\alpha})^{1 / 12}(1-\sqrt{1-\alpha})^{1 / 3} q^{-1 / 3} .
$$

Lastly, we consider $\chi\left(q^{n}\right)$, when $n$ is a power of 2 . Applying the process of duplication to $(2.12)$, we find that

$$
\chi\left(-q^{4}\right)=2^{5 / 12}(1-\alpha)^{1 / 48}(1+\sqrt{1-\alpha})^{1 / 12}(1-\sqrt{1-\alpha})^{-1 / 6} q^{1 / 6} .
$$


Invoking the duplication principle once again, but this time to (4.10), we find that

$\chi\left(-q^{8}\right)=2^{11 / 24}(1-\alpha)^{1 / 96}(1+\sqrt{1-\alpha})^{1 / 24}\left(1+(1-\alpha)^{1 / 4}\right)^{1 / 6}\left(1-(1-\alpha)^{1 / 4}\right)^{-1 / 3} q^{1 / 3}$.

Theorem 4.1. Let $S$ denote the set of partitions into nine distinct colors, with the red and blue parts appearing at most once with odd parts or parts in multiples of 8 . The remaining 7 colored parts appear at most once with parts congruent to 2 modulo 4. Let $T$ denote the set of partitions into six distinct colors, with the red, blue, green, and orange parts appearing at most once with odd parts or parts in multiples of 8 , and with the yellow and pink parts appearing at most once with parts congruent to 2 modulo 4. Let $D_{S}(N)$ denote the number of partitions of $N$ into distinct elements of $S$, and let $D_{T}(N)$ denote the number of partitions of $N$ into distinct elements of $T$. Then, for all $N \geq 1$,

$$
D_{S}(N)=2 D_{T}(N-1) \text {. }
$$

Proof. Utilizing elementary algebra, we can easily check that

$$
1+\sqrt{1-\alpha}=1-(1-\alpha)^{1 / 4}+(1-\alpha)^{1 / 4}\left\{1+(1-\alpha)^{1 / 4}\right\} .
$$

Dividing both sides of the identity $(4.12)$ by $(1-\alpha)^{1 / 4}\left\{1+(1-\alpha)^{1 / 4}\right\}$, we find that

$$
\frac{(1+\sqrt{1-\alpha})^{4 / 3}(1-\sqrt{1-\alpha})^{1 / 3}}{(1-\alpha)^{1 / 4} \alpha^{1 / 3}\left\{1+(1-\alpha)^{1 / 4}\right\}}=\frac{(1-\sqrt{1-\alpha})^{1 / 3}\left\{1-(1-\alpha)^{1 / 4}\right\}^{2 / 3}}{(1-\alpha)^{1 / 4}\left\{1+(1-\alpha)^{1 / 4}\right\}^{4 / 3}}+1 .
$$

The last identity can be expressed in the form

$$
\begin{aligned}
& \frac{(1-\alpha)^{5 / 24}(\alpha / q)^{-5 / 12}(1+\sqrt{1-\alpha})^{5 / 4}(1-\alpha)^{1 / 16}(1+\sqrt{1-\alpha})^{1 / 4}}{(1-\alpha)^{1 / 6}(\alpha / q)^{-1 / 12}(1-\alpha)^{1 / 24}(1+\sqrt{1-\alpha})^{1 / 6}(1-\sqrt{1-\alpha})^{-1 / 3} q^{1 / 3}(1-\alpha)^{5 / 16}\left\{1+(1-\alpha)^{1 / 4}\right\}} \\
= & q \frac{\{\alpha(1-\alpha) / q\}^{-1 / 12}(1-\alpha)^{1 / 16}(1+\sqrt{1-\alpha})^{1 / 4}}{(1-\alpha)^{1 / 6}(\alpha / q)^{-1 / 12}(1-\alpha)^{1 / 24}(1+\sqrt{1-\alpha})^{1 / 6}(1-\sqrt{1-\alpha})^{-1 / 3} q^{1 / 3}\left(1+(1-\alpha)^{1 / 4}\right)} \\
& \times \frac{1}{(1-\alpha)^{1 / 48}(1+\sqrt{1-\alpha})^{1 / 12}\left(1+(1-\alpha)^{1 / 4}\right)^{1 / 3}\left(1-(1-\alpha)^{1 / 4}\right)^{-2 / 3} q^{2 / 3}}+1 .
\end{aligned}
$$

Examining both side of (4.13), we apply (2.10), (2.11), (2.12), (4.2), (4.3), (4.5), (4.6), (4.10), and (4.11) to find that

$$
\frac{\chi^{5}\left(-q^{2}\right) \varphi^{5 / 2}\left(q^{2}\right) \varphi\left(-q^{4}\right)}{\chi^{2}(-q) \chi^{2}\left(-q^{4}\right) \varphi^{5 / 2}\left(-q^{2}\right) \varphi\left(q^{4}\right)}=2 q \frac{\chi^{2}(q) \varphi\left(-q^{4}\right)}{\chi^{2}(-q) \chi^{2}\left(-q^{4}\right) \varphi\left(q^{4}\right) \chi^{2}\left(-q^{8}\right)}+1 .
$$

Employing the identity

$$
\chi\left(q^{2}\right)=\chi\left(-q^{2}\right) \sqrt{\frac{\varphi\left(q^{2}\right)}{\varphi\left(-q^{2}\right)}}
$$


which is easily established from the product representations in (2.1) and (2.3), we find that (4.14) can be written in the form

$$
\frac{\chi^{5}\left(q^{2}\right)}{\chi^{2}(-q) \chi^{2}\left(q^{4}\right)}=2 q \frac{\chi^{2}(q)}{\chi^{2}(-q) \chi^{2}\left(q^{4}\right) \chi^{2}\left(-q^{8}\right)}+1 .
$$

Using the aforementioned representations of $\chi$ and $\varphi$, and Euler's identity (2.13), we obtain

$$
\frac{(-q ; q)_{\infty}^{2}\left(-q^{2} ; q^{4}\right)_{\infty}^{5}}{\left(-q^{4} ; q^{8}\right)_{\infty}^{2}}=2 q \frac{(-q ; q)_{\infty}^{2}\left(-q ; q^{2}\right)_{\infty}^{2}\left(-q^{8} ; q^{8}\right)_{\infty}^{2}}{\left(-q^{4} ; q^{8}\right)_{\infty}^{2}}+1 .
$$

Equating the coefficients of $q^{N}$ on both sides of this equation, we complete the proof.

Remark. Theorem 4.1 is equivalent to Conjecture 3.32 in Sandon and Zanello's paper [16].

Example 4.2. Let $N=4$ in Theorem 4.1. Then $D_{S}(4)=2^{2}+\left(\begin{array}{l}7 \\ 2\end{array}\right)+\left(\begin{array}{l}7 \\ 1\end{array}\right)=32$ and $D_{T}(3)=4+2 \cdot 4+\left(\begin{array}{l}4 \\ 3\end{array}\right)=16$. The relevant partitions are given by

$$
\begin{aligned}
& 3_{r}+1_{r}=\cdots=2_{1}+2_{7}=\cdots=2_{1}+1_{r}+1_{b}=\cdots ; \\
& 3_{r}=\cdots=3_{o}=2_{y}+1_{r}=\cdots=1_{r}+1_{b}+1_{g}=\cdots .
\end{aligned}
$$

Theorem 4.3. Let $S$ denote the set of partitions into six distinct colors, with the red, blue, green, and pink parts appearing at most once with odd parts or with parts congruent to 4 modulo 8, and the remaining two colors appearing at most once with the parts congruent to 2 modulo 4 . Let $T$ denote the set of partitions into nine distinct colors, with the red and blue parts appearing at most once with odd parts or in multiples of 8 , and the remaining seven colors appearing at most once with parts congruent to 2 modulo 4 . Let $D_{S}(N)$ denote the number of partitions of $N$ into an odd number of distinct elements of $S$. Let $D_{T}(N)$ be the number of partitions of $N$ into distinct elements of $T$. Then, for all $N \geq 1$,

$$
D_{S}(N)=D_{T}(N-1) \text {. }
$$

Proof. By elementary algebra, we can establish the identity

$$
\begin{aligned}
& \left\{\left(1+(1-\alpha)^{1 / 4}\right)^{2}-4(1-\alpha)^{3 / 4}\right\}\left(1+(1-\alpha)^{1 / 4}\right) \\
= & \left\{(1+\sqrt{1-\alpha})+3(1-\alpha)^{1 / 4}\left(1+(1-\alpha)^{1 / 4}\right)\right\}(1-\sqrt{1-\alpha}) .
\end{aligned}
$$

Multiplying both sides of the identity (4.16) by

we obtain

$$
\frac{2 q z_{1}^{3 / 2}(1-\alpha)^{3 / 8}}{\left(1+(1-\alpha)^{1 / 4}\right)(1-\sqrt{1-\alpha})}
$$

$$
\frac{2 q z_{1}^{3 / 2}(1-\alpha)^{3 / 8}}{1-\sqrt{1-\alpha}}\left\{\left(1+(1-\alpha)^{1 / 4}\right)^{2}-4(1-\alpha)^{3 / 4}\right\}
$$




$$
=\frac{2 q z_{1}^{3 / 2}(1-\alpha)^{3 / 8}(1+\sqrt{1-\alpha})}{1+(1-\alpha)^{1 / 4}}+6 q z_{1}^{3 / 2}(1-\alpha)^{5 / 8} .
$$

Examining first the left-hand side of (4.17), we call upon (2.6), (2.11), (2.12), (4.1), (4.2), (4.3), (4.5), (4.6), and (4.10) to find that, after considerable simplification,

$$
\begin{aligned}
& \frac{2 q z_{1}^{3 / 2}(1-\alpha)^{3 / 8}}{1-\sqrt{1-\alpha}}\left\{\left(1+(1-\alpha)^{1 / 4}\right)^{2}-4(1-\alpha)^{3 / 4}\right\} \\
= & \frac{\chi^{4}(-q) \chi^{4}\left(-q^{4}\right) \chi^{2}\left(-q^{2}\right)}{\varphi^{2}\left(-q^{4}\right)}\left\{\varphi^{2}(q) \varphi^{2}\left(q^{4}\right) \varphi\left(q^{2}\right)-\varphi^{2}(-q) \varphi^{2}\left(-q^{4}\right) \varphi\left(-q^{2}\right)\right\} .
\end{aligned}
$$

To evaluate the right-hand side of (4.17), we turn again to $(2.10),(2.12),(4.1)$, (4.2), (4.4), (4.5), and (4.9) to deduce that

$$
\begin{aligned}
& \frac{2 q z_{1}^{3 / 2}(1-\alpha)^{3 / 8}(1+\sqrt{1-\alpha})}{1+(1-\alpha)^{1 / 4}}+6 q z_{1}^{3 / 2}(1-\alpha)^{5 / 8} \\
= & \frac{2 q \chi^{2}(q) \chi^{7}\left(-q^{2}\right) f^{2}\left(-q^{8}\right) \varphi^{2}(-q) \varphi^{7 / 2}\left(q^{2}\right)}{\varphi^{2}\left(-q^{8}\right) \varphi^{5 / 2}\left(-q^{2}\right)}+6 q \varphi^{2}(-q) \varphi\left(-q^{2}\right) .
\end{aligned}
$$

Using the last two equalities in (4.17), we find that

$$
\begin{aligned}
& \frac{\chi^{4}(-q) \chi^{4}\left(-q^{4}\right) \chi^{2}\left(-q^{2}\right)}{\varphi^{2}\left(-q^{4}\right)}\left\{\varphi^{2}(q) \varphi^{2}\left(q^{4}\right) \varphi\left(q^{2}\right)-\varphi^{2}(-q) \varphi^{2}\left(-q^{4}\right) \varphi\left(-q^{2}\right)\right\} \\
= & \frac{2 q \chi^{2}(q) \chi^{7}\left(-q^{2}\right) f^{2}\left(-q^{8}\right) \varphi^{2}(-q) \varphi^{7 / 2}\left(q^{2}\right)}{\varphi^{2}\left(-q^{8}\right) \varphi^{5 / 2}\left(-q^{2}\right)}+6 q \varphi^{2}(-q) \varphi\left(-q^{2}\right) .
\end{aligned}
$$

Dividing both sides of (4.18) by $\varphi^{2}(-q) \varphi\left(-q^{2}\right)$ and using (4.15), we can simplify the last identity and write it in the shape

$$
\chi^{4}(q) \chi^{4}\left(q^{4}\right) \chi^{2}\left(q^{2}\right)-\chi^{4}(-q) \chi^{4}\left(-q^{4}\right) \chi^{2}\left(-q^{2}\right)=2 q \frac{\chi^{2}(q) \chi^{7}\left(q^{2}\right) f^{2}\left(-q^{8}\right)}{\varphi^{2}\left(-q^{8}\right)}+6 q .
$$

Applying the definitions of $\chi, f$, and $\varphi$, in (2.3), (2.2), and (2.1), respectively, we find that

$$
\begin{aligned}
& \left(-q ; q^{2}\right)_{\infty}^{4}\left(-q^{4} ; q^{8}\right)_{\infty}^{4}\left(-q^{2} ; q^{4}\right)_{\infty}^{2}-\left(q ; q^{2}\right)_{\infty}^{4}\left(q^{4} ; q^{8}\right)_{\infty}^{4}\left(q^{2} ; q^{4}\right)_{\infty}^{2} \\
= & 2 q\left(-q ; q^{2}\right)_{\infty}^{2}\left(-q^{8} ; q^{8}\right)_{\infty}^{2}\left(-q^{2} ; q^{4}\right)_{\infty}^{7}+6 q .
\end{aligned}
$$

Equating the coefficients of $q^{N}$ on both sides of the equation above, we finish the proof.

Remark. Theorem 4.3 is equivalent to Conjecture 3.33 in Sandon and Zanello's paper [16].

Example 4.4. Let $N=5$ in Theorem 4.3. Then $D_{S}(5)=4+4\left(\begin{array}{l}4 \\ 2\end{array}\right)+4=32$ and $D_{T}(4)=2^{2}+\left(\begin{array}{l}7 \\ 2\end{array}\right)+7=32$, with the associated partitions being given by

$$
5_{r}=5_{b}=5_{g}=5_{p}=3_{r}+1_{r}+1_{b}=\cdots=2_{w}+2_{o}+1_{r}=\cdots \text {; }
$$




$$
3_{r}+1_{r}=\cdots=2_{1}+2_{2}=\cdots=2_{1}+1_{r}+1_{b}=\cdots .
$$

Theorem 4.5. Let $S$ denote the set of partitions into fifteen distinct colors, with the red and blue parts appearing at most once with odd parts, with the green, orange, and pink parts appearing at most once with parts congruent to 2 modulo 4, with six further colors appearing at most once with parts congruent to 4 modulo 8, and with the remaining six colors appearing at most once with parts that are multiples of 8 . Let $T$ denote the set of partitions into fifteen distinct colors, with the red and blue parts appearing at most once with odd parts, with the green, orange, and pink parts appearing at most once with parts congruent to 2 modulo 4, with four additional colors appearing at most once with parts congruent to 4 modulo 8 , and with the remaining six colors appearing at most once with parts that are multiples of 8 . Let $D_{S}(N)$ denote the number of partitions of $N$ into distinct elements of $S$, and let $D_{T}(N)$ denote the number of partitions of $N$ into distinct elements of $T$. Then, for all $N \geq 1$,

$$
D_{S}(N)=2 D_{T}(N-1) \text {. }
$$

Proof. Utilizing elementary algebra, we can easily check that

$$
1+(1-\alpha)^{1 / 4}=\frac{\alpha^{1 / 6}\left(1-(1-\alpha)^{1 / 4}\right)(1-\sqrt{1-\alpha})^{1 / 6}}{(1+\sqrt{1-\alpha})^{1 / 6}(1-\sqrt{1-\alpha})^{1 / 3}}+2(1-\alpha)^{1 / 4}
$$

Dividing both sides of the identity $(4.19)$ by $2(1-\alpha)^{1 / 4}$ and performing extensive manipulation, we deduce that

$$
\begin{aligned}
& (4.20) \frac{(1-\alpha)^{1 / 48}(1+\sqrt{1-\alpha})^{1 / 12}(1-\sqrt{1-\alpha})^{-1 / 6} q^{1 / 6} \sqrt{z_{1}}\left(1+(1-\alpha)^{1 / 4}\right)}{2(1-\alpha)^{1 / 6}(\alpha / q)^{-1 / 12}(1-\alpha)^{1 / 24}(\alpha / q)^{-1 / 12} \sqrt{z_{1}}(1-\alpha)^{1 / 16}(1+\sqrt{1-\alpha})^{1 / 4}} \\
& =1+\frac{q}{2(1-\alpha)^{1 / 6}(\alpha / q)^{-1 / 12}(1-\alpha)^{1 / 24}(\alpha / q)^{-1 / 12}(1-\alpha)^{1 / 48}(1+\sqrt{1-\alpha})^{1 / 12}(1-\sqrt{1-\alpha})^{-1 / 6} q^{1 / 6}} \\
& \quad \times \frac{1}{(1-\alpha)^{1 / 48}(1+\sqrt{1-\alpha})^{1 / 12}\left(1+(1-\alpha)^{1 / 4}\right)^{1 / 3}\left(1-(1-\alpha)^{1 / 4}\right)^{-2 / 3} q^{2 / 3}} .
\end{aligned}
$$

Examining (4.20), we invoke (2.11), (2.12), (4.3), (4.6), (4.10), and (4.11) to find that

$$
\frac{\chi\left(-q^{4}\right) \varphi\left(q^{4}\right)}{\chi^{2}(-q) \chi\left(-q^{2}\right) \varphi\left(-q^{4}\right)}=1+\frac{2 q}{\chi^{2}(-q) \chi\left(-q^{2}\right) \chi\left(-q^{4}\right) \chi^{2}\left(-q^{8}\right)} .
$$

Applying (4.15) with $q$ replaced by $q^{2}$, we can rewrite the foregoing equation in the form

$$
\frac{\chi^{2}\left(q^{4}\right)}{\chi^{2}(-q) \chi\left(-q^{2}\right) \chi\left(-q^{4}\right)}=1+\frac{2 q}{\chi^{2}(-q) \chi\left(-q^{2}\right) \chi\left(-q^{4}\right) \chi^{2}\left(-q^{8}\right)} .
$$

Using the definition of $\chi$ from (2.3), and Euler's identity (2.13), we finally arrive at

$$
\begin{aligned}
& (-q ; q)_{\infty}^{2}\left(-q^{2} ; q^{2}\right)_{\infty}\left(-q^{4} ; q^{4}\right)_{\infty}\left(-q^{4} ; q^{8}\right)_{\infty}^{2} \\
= & 1+2 q(-q ; q)_{\infty}^{2}\left(-q^{2} ; q^{2}\right)_{\infty}\left(-q^{4} ; q^{4}\right)_{\infty}\left(-q^{8} ; q^{8}\right)_{\infty}^{2}
\end{aligned}
$$


Equating the coefficients of $q^{N}$ on both sides of the last equation, we finish the proof.

Remark. Theorem 4.5 is equivalent to Conjecture 3.35 in Sandon and Zanello's paper [16].

Example 4.6. Let $N=4$ in Theorem 4.5. Then $D_{S}(4)=6+2^{2}+\left(\begin{array}{l}3 \\ 2\end{array}\right)+3=16$ and $D_{T}(3)=2+3 \cdot 2=8$, with the relevant partitions being given by

$$
\begin{aligned}
& 4_{1}=\cdots=4_{6}=3_{r}+1_{r}=\cdots=2_{g}+2_{o}=\cdots=2_{g}+1_{r}+1_{b}=\cdots ; \\
& 3_{r}=3_{b}=2_{g}+1_{r}=\cdots .
\end{aligned}
$$

Theorem 4.7. Let $S$ denote the set of partitions into four distinct colors, with the red and blue parts appearing at most once, and with the green and pink parts appearing at most once with only odd parts. Let $T$ denote the set of partitions into fifteen distinct colors, with the red and blue parts appearing at most once with odd parts, with the green, orange, and pink parts appearing at most once with parts congruent to 2 modulo 4, with four further colors appearing at most once with parts congruent to 4 modulo 8, and with the remaining six colors appearing at most once with parts that are multiples of 8 . Let $D_{S}(N)$ denote the number of partitions of $N$ into distinct elements of $S$, and let $D_{T}(N)$ denote the number of partitions of $N$ into distinct elements of $T$. Then, for all $N \geq 1$,

$$
D_{S}(N)=4 D_{T}(N-1) \text {. }
$$

Proof. Applying elementary algebra, we can easily check that

$$
1-(1-\alpha)^{1 / 4}=\frac{\left(1-(1-\alpha)^{1 / 4}\right)(1-\sqrt{1-\alpha})^{1 / 6}}{\alpha^{-1 / 6}(1-\sqrt{1-\alpha})^{1 / 3}(1+\sqrt{1-\alpha})^{1 / 6}} .
$$

Dividing both sides of the identity $(4.21)$ by $(1-\alpha)^{1 / 4}$ and rearranging the resulting identity, we arrive at

$$
\frac{\{\alpha(1-\alpha) / q\}^{-1 / 12}}{(1-\alpha)^{1 / 6}(\alpha / q)^{-1 / 12}}
$$

$$
\begin{aligned}
= & 1+\frac{q}{(1-\alpha)^{1 / 6}(\alpha / q)^{-1 / 12}(1-\alpha)^{1 / 24}(\alpha / q)^{-1 / 12}(1-\alpha)^{1 / 48}(1+\sqrt{1-\alpha})^{1 / 12}(1-\sqrt{1-\alpha})^{-1 / 6} q^{1 / 6}} \\
& \times \frac{1}{(1-\alpha)^{1 / 48}(1+\sqrt{1-\alpha})^{1 / 12}\left(1+(1-\alpha)^{1 / 4}\right)^{1 / 3}\left(1-(1-\alpha)^{1 / 4}\right)^{-2 / 3} q^{2 / 3}} .
\end{aligned}
$$

Examining both side of (4.22), we call upon (2.10)-(2.12), (4.10), and (4.11) to find that,

$$
\frac{\chi^{2}(q)}{\chi^{2}(-q)}=1+\frac{4 q}{\chi^{2}(-q) \chi\left(-q^{2}\right) \chi\left(-q^{4}\right) \chi^{2}\left(-q^{8}\right)} .
$$

Using the definition of $\chi$ in (2.3), and Euler's identity (2.13), we deduce that

$$
(-q ; q)_{\infty}^{2}\left(-q ; q^{2}\right)_{\infty}^{2}=1+4 q(-q ; q)_{\infty}^{2}\left(-q^{2} ; q^{2}\right)_{\infty}\left(-q^{4} ; q^{4}\right)_{\infty}\left(-q^{8} ; q^{8}\right)_{\infty}^{2}
$$


Equating the coefficients of $q^{N}$ on both sides of the last equation, we complete the proof.

Remark. Theorem 4.7 is equivalent to Conjecture 3.36 in Sandon and Zanello's paper [16].

Example 4.8. Let $N=4$ in Theorem 4.7. Then $D_{S}(4)=2+4^{2}+1+2\left(\begin{array}{l}4 \\ 2\end{array}\right)+1=$ 32 and $D_{T}(3)=2+3 \cdot 2=8$, with the desired partitions being given by

$$
\begin{aligned}
& 4_{r}=4_{b}=3_{r}+1_{g}=\cdots=2_{r}+2_{b}=2_{r}+1_{g}+1_{p}=\cdots=1_{r}+1_{b}+1_{g}+1_{p} ; \\
& 3_{r}=3_{b}=2_{g}+1_{r}=\cdots .
\end{aligned}
$$

Theorem 4.9. Let $S$ denote the set of partitions into fifteen distinct colors, with the red and blue parts appearing at most once with odd parts, with the green, orange, and pink parts appearing at most once with parts congruent to 2 modulo 4, with six further colors appearing at most once with parts congruent to 4 modulo 8, and with the remaining four colors appearing at most once with parts in multiples of 8 . Let $T$ denote the set of partitions into four distinct colors, with the red and blue parts appearing at most once, and with the green and pink parts appearing at most once with only odd parts. If $D_{S}(N)$ denotes the number of partitions of $N$ into distinct elements of $S$, and if $D_{T}(N)$ denotes the number of partitions of $N$ into distinct elements of $T$, then, for all $N \geq 1$,

$$
D_{S}(N)=\frac{1}{2} D_{T}(N) \text {. }
$$

Proof. Utilizing elementary algebra, we can obtain the equation

$$
\frac{1+(1-\alpha)^{1 / 4}}{\alpha^{-1 / 6}(1+\sqrt{1-\alpha})^{1 / 6}(1-\sqrt{1-\alpha})^{1 / 6}}=1+(1-\alpha)^{1 / 4} .
$$

Dividing both sides of the identity $(4.23)$ by $(1-\alpha)^{1 / 4}$, we see that

$$
\begin{aligned}
& \frac{\left(1+(1-\alpha)^{1 / 4}\right)(1-\alpha)^{1 / 48}(1+\sqrt{1-\alpha})^{1 / 12}(1-\sqrt{1-\alpha})^{-1 / 6} q^{1 / 6}}{(1-\alpha)^{1 / 16}(1+\sqrt{1-\alpha})^{1 / 4}(1-\alpha)^{1 / 6}(\alpha / q)^{-1 / 12}(1-\alpha)^{1 / 24}(\alpha / q)^{-1 / 12}} \\
= & \frac{(\alpha(1-\alpha) / q)^{-1 / 12}}{(1-\alpha)^{1 / 6}(\alpha / q)^{-1 / 12}}+1 .
\end{aligned}
$$

To identify both sides of (4.24) in terms of theta functions, we turn again to (2.10)-(2.12), (4.3), (4.6), and (4.10) to deduce that

$$
\frac{2 \varphi\left(q^{4}\right) \chi\left(-q^{4}\right)}{\varphi\left(-q^{4}\right) \chi^{2}(-q) \chi\left(-q^{2}\right)}=\frac{\chi^{2}(q)}{\chi^{2}(-q)}+1 .
$$

Using (4.15) with $q$ replaced by $q^{2}$, we can simplify the last identity and find that

$$
\frac{2 \chi^{2}\left(q^{4}\right)}{\chi^{2}(-q) \chi\left(-q^{2}\right) \chi\left(-q^{4}\right)}=\frac{\chi^{2}(q)}{\chi^{2}(-q)}+1 \text {. }
$$


Applying the definition (2.3) of $\chi$ and invoking Euler's identity (2.13), we find that

$$
2(-q ; q)_{\infty}^{2}\left(-q^{2} ; q^{2}\right)_{\infty}\left(-q^{4} ; q^{4}\right)_{\infty}\left(-q^{4} ; q^{8}\right)_{\infty}^{2}=(-q ; q)_{\infty}^{2}\left(-q ; q^{2}\right)_{\infty}^{2}+1
$$

Equating the coefficients of $q^{N}$ on both sides of this equation, we finish the proof.

Remark. Theorem 4.9 is equivalent to Conjecture 3.37 in Sandon and Zanello's paper [16].

Example 4.10. Let $N=4$ in Theorem 4.9. Then $D_{S}(4)=6+2^{2}+\left(\begin{array}{l}3 \\ 2\end{array}\right)+3=16$ and $D_{T}(4)=2+4^{2}+1+2\left(\begin{array}{l}4 \\ 2\end{array}\right)+1=32$; the desired partitions are given by

$$
\begin{aligned}
& 4_{1}=\cdots=4_{6}=3_{r}+1_{r}=\cdots=2_{g}+2_{o}=\cdots=2_{g}+1_{r}+1_{b} ; \\
& 4_{r}=4_{b}=3_{r}+1_{g}=\cdots=2_{r}+2_{b}=2_{r}+1_{g}+1_{p}=\cdots=1_{r}+1_{b}+1_{g}+1_{p} .
\end{aligned}
$$

Theorem 4.11. Let $S$ denote the set of partitions into eight distinct colors, with the red, blue, and yellow parts appearing at most once with even parts, with the green parts appearing at most once with odd parts, with three additional colors appearing at most once with odd parts in multiples of 5 , and with one last color appearing at most once with parts in multiples of 10 . Let $T$ denote the set of partitions into eight distinct colors, with the red, blue, and yellow parts appearing at most once with odd parts, with the green parts appearing at most once with even parts, with one further color appearing at most once with odd parts in multiples of 5, and with parts in 3 additional colors parts appearing at most once with parts in multiples of 10 . If $D_{S}(N)$ denotes the number of partitions of $N$ into distinct elements of $S$, and if $D_{T}(N)$ denotes the number of partitions of $N$ into distinct elements of $T$, then, for all $N \geq 1$,

$$
D_{S}(N)=D_{T}(N-1) \text {. }
$$

Proof. First we recall the parameterizations [7, p. 284, equations (13.4), (13.5)], $[7$, p. 285, equations $(13.10),(13.11)]$, and [7, p. 286, equations (13.11)]

$$
\begin{gathered}
\left(\frac{\alpha^{5}}{\beta}\right)^{1 / 8}=\frac{5 \rho+m^{2}+5 m}{4 m^{2}}, \quad\left(\frac{\beta^{5}}{\alpha}\right)^{1 / 8}=\frac{\rho-m-1}{4}, \\
\left(\frac{(1-\beta)^{5}}{1-\alpha}\right)^{1 / 8}=\frac{\rho+m+1}{4}, \quad\left(\frac{(1-\alpha)^{5}}{1-\beta}\right)^{1 / 8}=\frac{5 \rho-m^{2}-5 m}{4 m^{2}}, \\
\left(\alpha^{3} \beta\right)^{1 / 8}=\frac{\rho+3 m-5}{4 m}, \quad\left(\alpha \beta^{3}\right)^{1 / 8}=\frac{\rho+m^{2}-3 m}{4 m},
\end{gathered}
$$

where $\beta$ has degree 5 over $\alpha$ and $\rho=\left(m^{3}-2 m^{2}+5 m\right)^{1 / 2}$. Indeed, by simple elementary algebra, we can find that

$$
\left(\frac{\alpha^{5}}{\beta}\right)^{1 / 8}-3\left(\alpha^{3} \beta\right)^{1 / 8}+3\left(\alpha \beta^{3}\right)^{1 / 8}-\left(\frac{\beta^{5}}{\alpha}\right)^{1 / 8}=4(1-\alpha)^{1 / 2}(1-\beta)^{1 / 2}
$$


Next, extract the real cube root on each side of (4.25) to obtain the equation

$$
\left(\frac{\alpha^{5}}{\beta}\right)^{1 / 24}-\left(\frac{\beta^{5}}{\alpha}\right)^{1 / 24}=2^{2 / 3}(1-\alpha)^{1 / 6}(1-\beta)^{1 / 6}
$$

Divide both sides of $(4.26)$ by $2^{2 / 3}(1-\alpha)^{1 / 6}(1-\beta)^{1 / 6}$ to achieve the resulting identity

$$
\begin{aligned}
& \frac{(1-\beta)^{1 / 12}\left(\beta / q^{5}\right)^{-1 / 6}}{2^{2 / 3}(1-\alpha)^{1 / 12}(\alpha / q)^{-1 / 24}(1-\alpha)^{1 / 12}(\alpha / q)^{-1 / 6}(1-\beta)^{1 / 4}\left(\beta / q^{5}\right)^{-1 / 8}} \\
= & q \frac{\{\alpha(1-\alpha) / q\}^{-1 / 12}}{2^{2 / 3}(1-\alpha)^{1 / 12}(\alpha / q)^{-1 / 24}(1-\beta)^{1 / 4}\left(\beta / q^{5}\right)^{-1 / 8}\left\{\beta(1-\beta) / q^{5}\right\}^{-1 / 12}}+1 .
\end{aligned}
$$

Applying (2.10)-(2.12) from Lemma 2.1 in the last identity, we deduce that

$$
\frac{\chi^{2}\left(-q^{10}\right)}{\chi(-q) \chi^{2}\left(-q^{2}\right) \chi^{3}\left(-q^{5}\right)}=q \frac{\chi^{2}(q)}{\chi(-q) \chi^{3}\left(-q^{5}\right) \chi^{2}\left(q^{5}\right)}+1 \text {. }
$$

Applying the definition of $\chi$ in (2.3), and Euler's identity (2.13), we obtain the $q$-product version of the foregoing equation, namely,

$$
\frac{(-q ; q)_{\infty}\left(-q^{2} ; q^{2}\right)_{\infty}^{2}\left(-q^{5} ; q^{5}\right)_{\infty}^{3}}{\left(-q^{10} ; q^{10}\right)_{\infty}^{2}}=q \frac{(-q ; q)_{\infty}\left(-q ; q^{2}\right)_{\infty}^{2}\left(-q^{5} ; q^{5}\right)_{\infty}^{3}}{\left(-q^{5} ; q^{10}\right)_{\infty}^{2}}+1
$$

Equate the coefficients of $q^{N}$ on both sides of this equation to complete the proof.

Remark. Theorem 4.11 is equivalent to Conjecture 3.40 in Sandon and Zanello's paper [16].

Example 4.12. Let $N=5$ in Theorem 4.11. Then $D_{S}(5)=4+3+3+3=13$ and $D_{T}(4)=1+3^{2}+3=13$, with the desired partitions being given by

$$
\begin{aligned}
& 5_{g}=\cdots=5_{3}=4_{r}+1_{g}=\cdots=3_{g}+2_{b}=\cdots=2_{r}+2_{y}+1_{g}=\cdots ; \\
& 4_{g}=3_{r}+1_{b}=\cdots=2_{g}+1_{r}+1_{y}=\cdots .
\end{aligned}
$$

\section{Proofs of twelve conjectures using modular equations of degree 3}

Theorem 5.1. Let $S$ denote the set of partitions into twelve distinct colors, with the red parts appearing at most once with parts congruent to \pm 1 modulo 6 , with five colors appearing at most once with parts congruent to \pm 2 modulo 6 , and with six colors appearing at most once with parts in multiples of 3 . Let $T$ denote the set of partitions into twelve distinct colors, with the red parts appearing at most once with parts congruent to \pm 2 modulo 6 , with five colors appearing at most once with parts congruent to \pm 1 modulo 6 , and with six colors appearing at most once with parts in multiples of 3 . If $D_{S}(N)$ denotes the number of partitions of $N$ into distinct elements of $S$ and if $D_{T}(N)$ denotes the number of partitions of $N$ into distinct elements of $T$, then, for all $N \geq 1$,

$$
D_{S}(N)=D_{T}(N-1) \text {. }
$$


Proof. First we recall the parameterizations [7, p. 232, equations (5.1)]

$$
\begin{array}{ll}
\left(\frac{\beta^{3}}{\alpha}\right)^{1 / 8}=\frac{m-1}{2}, & \left(\frac{(1-\beta)^{3}}{1-\alpha}\right)^{1 / 8}=\frac{m+1}{2}, \\
\left(\frac{\alpha^{3}}{\beta}\right)^{1 / 8}=\frac{3+m}{2 m}, & \left(\frac{(1-\alpha)^{3}}{1-\beta}\right)^{1 / 8}=\frac{3-m}{2 m},
\end{array}
$$

where $\beta$ has degree 3 over $\alpha$. Indeed, by simple elementary algebra, we can find that

$$
\left(\frac{\alpha^{3}}{\beta}\right)^{1 / 8}-\left(\frac{\beta^{3}}{\alpha}\right)^{1 / 8}=2(1-\alpha)^{1 / 4}(1-\beta)^{1 / 4}
$$

Next, divide both sides of $(5.1)$ by $2(1-\alpha)^{1 / 4}(1-\beta)^{1 / 4}$ to obtain the equation

$$
\begin{aligned}
& \frac{(1-\beta)^{1 / 6}\left(\beta / q^{3}\right)^{-1 / 3}}{2(1-\alpha)^{1 / 12}(\alpha / q)^{-1 / 24}(1-\alpha)^{1 / 6}(\alpha / q)^{-1 / 3}(1-\beta)^{5 / 12}\left(\beta / q^{3}\right)^{-5 / 24}} \\
= & q \frac{\{\alpha(1-\alpha) / q\}^{-1 / 6}}{2(1-\alpha)^{1 / 12}(\alpha / q)^{-1 / 24}(1-\beta)^{1 / 12}\left(\beta / q^{3}\right)^{-1 / 24}(1-\beta)^{1 / 6}\left(\beta / q^{3}\right)^{-1 / 3}}+1 .
\end{aligned}
$$

From (2.10)-(2.12) in Lemma 2.1, we find that the foregoing identity can be written as

$$
\frac{\chi^{4}\left(-q^{6}\right)}{\chi(-q) \chi^{4}\left(-q^{2}\right) \chi^{5}\left(-q^{3}\right)}=q \frac{\chi^{4}(q)}{\chi(-q) \chi\left(-q^{3}\right) \chi^{4}\left(-q^{6}\right)}+1
$$

Hence, applying the definition of $\chi$ in (2.3), we obtain the $q$-product form of the last identity, namely,

$$
\frac{(-q ; q)_{\infty}\left(-q^{2} ; q^{2}\right)_{\infty}^{4}\left(-q^{3} ; q^{3}\right)_{\infty}^{5}}{\left(-q^{6} ; q^{6}\right)_{\infty}^{4}}=q(-q ; q)_{\infty}\left(-q ; q^{2}\right)_{\infty}^{4}\left(-q^{3} ; q^{3}\right)_{\infty}\left(-q^{6} ; q^{6}\right)_{\infty}^{4}+1
$$

Equating the coefficients of $q^{N}$ on both sides of this equation, we finish the proof.

Remark. Theorem 5.1 is equivalent to Conjecture 3.34 in Sandon and Zanello's paper [16].

Example 5.2. Let $N=4$ in Theorem 5.1. Then $D_{S}(4)=5+6+\left(\begin{array}{l}5 \\ 2\end{array}\right)=21$ and $D_{T}(3)=6+5+\left(\begin{array}{l}5 \\ 3\end{array}\right)=21$, with the relevant partitions being given by

$$
\begin{aligned}
& 4_{1}=4_{2}=\cdots=4_{5}=3_{6}+1_{r}=\cdots=2_{1}+2_{5}=\cdots ; \\
& 3_{1}=\cdots=3_{6}=2_{r}+1_{5}=\cdots=1_{1}+1_{2}+1_{3}=\cdots .
\end{aligned}
$$

Theorem 5.3. Let $S$ denote the set of partitions into four distinct colors, with the red and blue parts appearing at most once without multiples of 4, and the green and pink parts appearing at most once with parts in multiples of 3 , but not multiples of 4 . Let $T$ denote the set of partitions into four distinct colors, with the red and blue parts appearing at most once without parts congruent to 2 modulo 4, and the green and pink colors appearing at most once with parts in multiples of 3 , but not congruent to 2 modulo 4 . Let $D_{S}(N)$ denote the number 
of partitions of $N$ into an odd number of distinct elements of $S$, and let $D_{T}(N)$ denote the number of partitions of $N$ into distinct elements of $T$. Then, for all $N \geq 2$,

$$
D_{S}(N)=2 D_{T}(N-2) \text {. }
$$

Proof. First we recall the parameterizations [7, p. 233, equations (5.2), (5.5)]

$$
\begin{array}{cc}
\alpha=\frac{(m-1)(3+m)^{3}}{16 m^{3}}, & \beta=\frac{(m-1)^{3}(3+m)}{16 m}, \\
1-\alpha=\frac{(m+1)(3-m)^{3}}{16 m^{3}}, & 1-\beta=\frac{(m+1)^{3}(3-m)}{16 m},
\end{array}
$$

where $\beta$ has degree 3 over $\alpha$. By simple elementary algebra, we can check that (5.4)

$1+\sqrt{(1-\alpha)(1-\beta)}-\sqrt{\alpha \beta}=2\{(1-\alpha)(1-\beta)\}^{1 / 4}\left\{\{(1-\alpha)(1-\beta)\}^{1 / 4}+(\alpha \beta)^{1 / 4}\right\}^{2}$.

Next, multiply both sides of (5.4) by 2 and extract the square root on both sides of the resulting identity to obtain the equation

$$
\begin{aligned}
& (1+\sqrt{1-\alpha})^{1 / 2}(1+\sqrt{1-\beta})^{1 / 2}-(1-\sqrt{1-\alpha})^{1 / 2}(1-\sqrt{1-\beta})^{1 / 2} \\
= & 2\{(1-\alpha)(1-\beta)\}^{1 / 8}\left\{\{(1-\alpha)(1-\beta)\}^{1 / 4}+(\alpha \beta)^{1 / 4}\right\} .
\end{aligned}
$$

Rearranging terms and multiplying both sides of $(5.5)$ by $(\alpha \beta)^{1 / 4}$, we arrive at

$$
\begin{aligned}
& (\alpha \beta)^{1 / 4}(1+\sqrt{1-\alpha})^{1 / 2}(1+\sqrt{1-\beta})^{1 / 2}-2(\alpha \beta)^{1 / 4}\{(1-\alpha)(1-\beta)\}^{3 / 8} \\
= & (\alpha \beta)^{1 / 4}(1-\sqrt{1-\alpha})^{1 / 2}(1-\sqrt{1-\beta})^{1 / 2}+2(\alpha \beta)^{1 / 2}\{(1-\alpha)(1-\beta)\}^{1 / 8} .
\end{aligned}
$$

Multiply both side of $(5.6)$ by $\frac{2 q}{(\alpha \beta)^{1 / 2}\{(1-\alpha)(1-\beta)\}^{1 / 8}}$. Hence,

$$
\begin{aligned}
& \frac{2 q(\alpha \beta)^{1 / 4}}{\{(1-\alpha)(1-\beta)\}^{1 / 8}(1-\sqrt{1-\alpha})^{1 / 2}(1-\sqrt{1-\beta})^{1 / 2}}-4 q\left(\frac{(1-\alpha)(1-\beta)}{\alpha \beta}\right)^{1 / 4} \\
= & 2 q \frac{(\alpha \beta)^{1 / 4}}{\{(1-\alpha)(1-\beta)\}^{1 / 8}(1+\sqrt{1-\alpha})^{1 / 2}(1+\sqrt{1-\beta})^{1 / 2}}+4 q .
\end{aligned}
$$

First, from (2.8) and (4.8),

$$
\frac{f^{2}(-q) f^{2}\left(-q^{3}\right)}{f^{2}\left(-q^{4}\right) f^{2}\left(-q^{12}\right)}=4 q\left(\frac{(1-\alpha)(1-\beta)}{\alpha \beta}\right)^{1 / 4} .
$$

Second, from (2.8), (4.1), (4.3), and (4.8),

$$
\begin{aligned}
& \frac{f^{2}(-q) f^{2}\left(-q^{3}\right)}{f^{2}\left(-q^{4}\right) f^{2}\left(-q^{12}\right)} \frac{\varphi^{2}\left(-q^{4}\right) \varphi^{2}\left(-q^{12}\right)}{\varphi^{2}(-q) \varphi^{2}\left(-q^{3}\right)} \\
= & \frac{2 q(\alpha \beta)^{1 / 4}}{\{(1-\alpha)(1-\beta)\}^{1 / 8}(1-\sqrt{1-\alpha})^{1 / 2}(1-\sqrt{1-\beta})^{1 / 2}} .
\end{aligned}
$$


Third, from (2.8), (2.12), (4.1), (4.2), (4.5), and (4.15),

$$
\begin{aligned}
& 4 q^{2} \frac{f^{2}(-q) f^{2}\left(-q^{3}\right)}{\varphi^{2}(-q) \varphi^{2}\left(-q^{3}\right) \chi^{2}\left(q^{2}\right) \chi^{2}\left(q^{6}\right)} \\
= & 4 q^{2} \frac{f^{2}(-q) f^{2}\left(-q^{3}\right) \varphi\left(-q^{2}\right) \varphi\left(-q^{6}\right)}{\varphi^{2}(-q) \varphi^{2}\left(-q^{3}\right) \chi^{2}\left(-q^{2}\right) \chi^{2}\left(-q^{6}\right) \varphi\left(q^{2}\right) \varphi\left(q^{6}\right)} \\
= & 2 q \frac{(\alpha \beta)^{1 / 4}}{\{(1-\alpha)(1-\beta)\}^{1 / 8}(1+\sqrt{1-\alpha})^{1 / 2}(1+\sqrt{1-\beta})^{1 / 2}} .
\end{aligned}
$$

Hence, from (5.7) and (5.8)-(5.10), it suffices to prove that

$(5.11)$

$$
\frac{f^{2}(-q) f^{2}\left(-q^{3}\right)}{f^{2}\left(-q^{4}\right) f^{2}\left(-q^{12}\right)}\left(\frac{\varphi^{2}\left(-q^{4}\right) \varphi^{2}\left(-q^{12}\right)}{\varphi^{2}(-q) \varphi^{2}\left(-q^{3}\right)}-1\right)=4 q^{2} \frac{f^{2}(-q) f^{2}\left(-q^{3}\right)}{\varphi^{2}(-q) \varphi^{2}\left(-q^{3}\right) \chi^{2}\left(q^{2}\right) \chi^{2}\left(q^{6}\right)}+4 q .
$$

Applying the definitions of $\varphi, f$, and $\chi$ from (2.1), (2.2), and (2.3), respectively, we can convert (5.11) into $q$-products, namely,

$$
\begin{aligned}
& \frac{(-q ; q)_{\infty}^{2}\left(-q^{3} ; q^{3}\right)_{\infty}^{2}}{\left(-q^{4} ; q^{4}\right)_{\infty}^{2}\left(-q^{12} ; q^{12}\right)_{\infty}^{2}}-\frac{(q ; q)_{\infty}^{2}\left(q^{3} ; q^{3}\right)_{\infty}^{2}}{\left(q^{4} ; q^{4}\right)_{\infty}^{2}\left(q^{12} ; q^{12}\right)_{\infty}^{2}} \\
= & 4 q^{2} \frac{(-q ; q)_{\infty}^{2}\left(-q^{3} ; q^{3}\right)_{\infty}^{2}}{\left(-q^{2} ; q^{4}\right)_{\infty}^{2}\left(-q^{6} ; q^{12}\right)_{\infty}^{2}}+4 q .
\end{aligned}
$$

Equating the coefficients of $q^{N}$ on both sides of the last equation, we finish the proof.

Remark. Theorem 5.3 is equivalent to Conjecture 3.42 in Sandon and Zanello's paper [16].

Example 5.4. Let $N=5$ in Theorem 5.3. Then $D_{S}(5)=2+4+2=8$ and $D_{T}(3)=4$. The partitions that we want are given by

$$
\begin{aligned}
& 5_{r}=5_{b}=3_{r}+1_{r}+1_{b}=3_{g}+1_{r}+1_{b}=\cdots=2_{r}+2_{b}+1_{r}=2_{r}+2_{b}+1_{b} ; \\
& 3_{r}=3_{b}=3_{g}=3_{p} .
\end{aligned}
$$

Theorem 5.5. Let $S$ denote the set of partitions into five distinct colors, with the red and blue parts appearing at most once without odd multiples of 3 , the green color appearing at most once with parts congruent to \pm 2 modulo 12, and the pink and orange colors appearing at most once with odd multiples of 6 . Let $T$ denote the set of partitions into five distinct colors, with the red and blue parts appearing at most once but not in odd multiples of 3, the green color appearing at most once with parts congruent to \pm 4 modulo 12 , and the pink and orange colors appearing at most once with parts in multiples of 12 . Let $D_{S}(N)$ denote the number of partitions of $N$ into distinct elements of $S$, and let $D_{T}(N)$ denote the number of partitions of $N$ into distinct elements of $T$. Then, for all $N \geq 1$,

$$
D_{S}(N)=2 D_{T}(N-1)
$$


Proof. Recall the parameterizations for $\alpha$ and $\beta$ of degree 3 given in (5.2) and (5.3). By simple elementary algebra, we can check that

$$
\begin{aligned}
& (1+\sqrt{1-\alpha})(1+\sqrt{1-\beta})+(1-\sqrt{1-\alpha})(1-\sqrt{1-\beta})+2 \alpha^{1 / 2} \beta^{1 / 2} \\
= & \left(\frac{m^{2}+3}{2 m}\right)^{2}=\left\{2 \alpha^{1 / 4} \beta^{1 / 4}+2\left\{\frac{(1-\alpha)^{3}}{1-\beta}\right\}^{1 / 8}\right\}^{2} .
\end{aligned}
$$

Next, extract the square root on both sides of the last identity and rearrange the resulting identity to obtain the equation

$$
\begin{aligned}
& (1+\sqrt{1-\alpha})^{1 / 2}(1+\sqrt{1-\beta})^{1 / 2}+(1-\sqrt{1-\alpha})^{1 / 2}(1-\sqrt{1-\beta})^{1 / 2} \\
& -2 \alpha^{1 / 4} \beta^{1 / 4} \\
= & 2\left\{\frac{(1-\alpha)^{3}}{1-\beta}\right\}^{1 / 8} .
\end{aligned}
$$

Extracting the square root on both sides of (5.12) and rearranging the resulting identity, we find that

$$
(1+\sqrt{1-\alpha})^{1 / 4}(1+\sqrt{1-\beta})^{1 / 4}=(1-\sqrt{1-\alpha})^{1 / 4}(1-\sqrt{1-\beta})^{1 / 4}+2^{1 / 2}\left\{\frac{(1-\alpha)^{3}}{1-\beta}\right\}^{1 / 16} .
$$

Dividing both sides of $(5.13)$ by $2^{1 / 2}\left\{\frac{(1-\alpha)^{3}}{1-\beta}\right\}^{1 / 16}$, and rewriting the resulting identity, we arrive at

$$
\begin{aligned}
& \frac{(1-\alpha)^{1 / 24}(\alpha / q)^{-1 / 12} z_{1}^{1 / 4}(1+\sqrt{1-\alpha})^{1 / 4}(1-\beta)^{1 / 24}\left(\beta / q^{3}\right)^{-1 / 12} z_{3}^{1 / 4}(1+\sqrt{1-\beta})^{1 / 4}}{2^{1 / 2}(1-\alpha)^{1 / 6}(\alpha / q)^{-1 / 12} z_{1}^{1 / 4}(1-\alpha)^{1 / 16}\left\{\beta(1-\beta) / q^{3}\right\}^{-1 / 12} z_{3}^{1 / 4}(1-\beta)^{1 / 16}} \\
= & \frac{q}{2^{1 / 2}(1-\alpha)^{1 / 6}(\alpha / q)^{-1 / 12}(1-\alpha)^{1 / 48}(1+\sqrt{1-\alpha})^{1 / 12}(1-\sqrt{1-\alpha})^{-1 / 6} q^{1 / 6}} \\
& \times \frac{1}{(1-\beta)^{1 / 48}(1+\sqrt{1-\beta})^{1 / 12}(1-\sqrt{1-\beta})^{-1 / 6} q^{1 / 2}\left\{\beta(1-\beta) / q^{3}\right\}^{-1 / 12}}+1 .
\end{aligned}
$$

Hence, from (2.10), (2.11), (2.12), (4.2), (4.5), and (4.10), it suffices to prove that

$$
\frac{\chi\left(-q^{2}\right) \sqrt{\varphi\left(q^{2}\right)} \chi\left(-q^{6}\right) \sqrt{\varphi\left(q^{6}\right)}}{\chi^{2}(-q) \sqrt{\varphi\left(-q^{2}\right)} \chi^{2}\left(q^{3}\right) \sqrt{\varphi\left(-q^{6}\right)}}=\frac{2 q}{\chi^{2}(-q) \chi\left(-q^{4}\right) \chi\left(-q^{12}\right) \chi^{2}\left(q^{3}\right)}+1 .
$$

Using the formula (4.15), we find that

$$
\frac{\chi\left(q^{2}\right) \chi\left(q^{6}\right)}{\chi^{2}(-q) \chi^{2}\left(q^{3}\right)}=\frac{2 q}{\chi^{2}(-q) \chi\left(-q^{4}\right) \chi\left(-q^{12}\right) \chi^{2}\left(q^{3}\right)}+1
$$

Applying the definitions of $\chi$ from (2.3), we can convert the last equation into $q$-products, namely,

$$
\frac{(-q ; q)_{\infty}^{2}\left(-q^{2} ; q^{4}\right)_{\infty}\left(-q^{6} ; q^{12}\right)_{\infty}}{\left(-q^{3} ; q^{6}\right)_{\infty}^{2}}=2 q \frac{(-q ; q)_{\infty}^{2}\left(-q^{4} ; q^{4}\right)_{\infty}\left(-q^{12} ; q^{12}\right)_{\infty}}{\left(-q^{3} ; q^{6}\right)_{\infty}^{2}}+1
$$


Equating the coefficients of $q^{N}$ on both sides of the last equation, we finish the proof.

Remark. Theorem 5.5 is equivalent to Conjecture 3.46 in Sandon and Zanello's paper [16].

Example 5.6. Let $N=5$ in Theorem 5.5. Then $D_{S}(5)=2+2^{2}+\left(\begin{array}{l}3 \\ 2\end{array}\right) \cdot 2=12$ and $D_{T}(4)=6$. The partitions that we want are given by

$$
\begin{aligned}
& 5_{r}=5_{b}=4_{r}+1_{b}=\cdots=2_{r}+2_{g}+1_{r}=\cdots ; \\
& 4_{r}=4_{b}=4_{g}=2_{r}+2_{b}=2_{r}+1_{r}+1_{b}=2_{b}+1_{r}+1_{b} .
\end{aligned}
$$

Theorem 5.7. Let $S$ denote the set of partitions into nine distinct colors, with the red and blue parts appearing at most once with multiples of 6 , the green and pink colors appearing at most once with parts congruent to \pm 1 modulo 6 , the orange color appearing at most once with parts congruent to \pm 2 modulo 6 , and the remaining four colors appearing at most once with parts that are odd multiples of 3. Let $T$ denote the set of partitions into ten distinct colors, with the red, blue, green, and pink parts appearing at most once in multiples of 6 , the orange color appearing at most once with parts congruent to \pm 1 modulo 6 , the yellow color appearing at most once with parts congruent to \pm 2 modulo 6 , another two colors appearing at most once with parts congruent to \pm 2 modulo 12 , and the remaining two colors appearing at most once with parts that are odd multiples of 3 . Let $D_{S}(N)$ denote the number of partitions of $N$ into distinct elements of $S$, and let $D_{T}(N)$ denote the number of partitions of $N$ into distinct elements of $T$. Then, for all $N \geq 1$,

$$
D_{S}(N)=2 D_{T}(N-1) \text {. }
$$

Proof. We again recall the parameterizations for $\alpha$ and $\beta$ given in (5.2) and (5.3). By simple elementary algebra, we can find that

$$
\begin{aligned}
& (1+\sqrt{1-\alpha})(1-\sqrt{1-\beta}) \\
= & \frac{-m^{4}+4 m^{3}+18 m^{2}-12 m-9}{16 m^{2}}-\frac{(m+3)(m-1) \sqrt{(m+1)(3-m)}}{4 m \sqrt{m}} \\
= & \alpha^{1 / 4} \beta^{1 / 4}+\{\alpha \beta(1-\alpha)(1-\beta)\}^{1 / 4}-2 \alpha^{1 / 4} \beta^{1 / 4}\{(1-\alpha)(1-\beta)\}^{1 / 8} .
\end{aligned}
$$

Extract the square root on both sides of the resulting identity and rearrange terms to obtain the equation

$$
\alpha^{1 / 8} \beta^{1 / 8}=(1+\sqrt{1-\alpha})^{1 / 2}(1-\sqrt{1-\beta})^{1 / 2}+\{\alpha \beta(1-\alpha)(1-\beta)\}^{1 / 8} .
$$

Dividing both sides of $(5.14)$ by $\{\alpha \beta(1-\alpha)(1-\beta)\}^{1 / 8}$ and rearranging terms, we arrive at

$$
\begin{aligned}
& \frac{\{\alpha(1-\alpha) / q\}^{-1 / 24}\left\{\beta(1-\beta) / q^{3}\right\}^{-1 / 24}}{(1-\alpha)^{1 / 12}(\alpha / q)^{-1 / 24}(1-\beta)^{1 / 12}\left(\beta / q^{3}\right)^{-1 / 24}} \\
= & q \frac{(1-\alpha)^{1 / 12}(\alpha / q)^{-1 / 6} \sqrt{z_{1}}(1+\sqrt{1-\alpha})^{1 / 2}}{(1-\alpha)^{1 / 12}(\alpha / q)^{-1 / 24}(1-\beta)^{1 / 12}\left(\beta / q^{3}\right)^{-1 / 24}}
\end{aligned}
$$




$$
\times \frac{1}{(1-\beta)^{1 / 24}(1+\sqrt{1-\beta})^{1 / 6}(1-\sqrt{1-\beta})^{-1 / 3} q \sqrt{z_{1}}(1-\alpha)^{1 / 8}}+1,
$$

which can be transformed into

$$
\frac{\chi(q) \chi\left(q^{3}\right)}{\chi(-q) \chi\left(-q^{3}\right)}=2 q \frac{\chi^{2}\left(-q^{2}\right) \varphi\left(q^{2}\right)}{\chi(-q) \chi\left(-q^{3}\right) \chi^{2}\left(-q^{12}\right) \varphi\left(-q^{2}\right)}+1
$$

by $(2.10),(2.11),(2.12),(4.2),(4.5)$, and (4.10). Applying (4.15), we check that

$$
\frac{\chi(q) \chi\left(q^{3}\right)}{\chi(-q) \chi\left(-q^{3}\right)}=2 q \frac{\chi^{2}\left(q^{2}\right)}{\chi(-q) \chi\left(-q^{3}\right) \chi^{2}\left(-q^{12}\right)}+1 .
$$

Applying the definitions of $\chi$ from (2.3), we can reformulate the last equation and deduce that

$$
\begin{aligned}
& \left(-q ; q^{2}\right)_{\infty}^{2}\left(-q^{2} ; q^{2}\right)_{\infty}\left(-q^{3} ; q^{6}\right)_{\infty}^{2}\left(-q^{6} ; q^{6}\right)_{\infty} \\
= & 2 q(-q ; q)_{\infty}\left(-q^{3} ; q^{3}\right)_{\infty}\left(-q^{2} ; q^{4}\right)_{\infty}^{2}\left(-q^{12} ; q^{12}\right)_{\infty}^{2}+1 .
\end{aligned}
$$

Equating the coefficients of $q^{N}$ on both sides of the last equation, we finish the proof.

Remark. Theorem 5.7 is equivalent to Conjecture 3.49 in Sandon and Zanello's paper [16].

Example 5.8. Let $N=4$ in Theorem 5.7. Then $D_{S}(4)=1+4 \cdot 2+1=10$ and $D_{T}(3)=5$. The partitions for this example are given by

$$
\begin{aligned}
& 4_{o}=3_{1}+1_{g}=3_{1}+1_{p}=\cdots=3_{4}+1_{p}=\cdots=2_{o}+1_{g}+1_{p} ; \\
& 3_{3}=3_{4}=2_{y}+1_{o}=2_{1}+1_{o}=2_{2}+1_{o} .
\end{aligned}
$$

Theorem 5.9. Let $S$ denote the set of partitions into ten distinct colors, with the red, blue, green, and pink parts appearing at most once in multiples of 6 , the orange color appearing at most once with parts congruent to \pm 1 modulo 6 , the yellow color appearing at most once with parts congruent to \pm 2 modulo 6 , another two colors appearing at most once with parts congruent to \pm 4 modulo 12, and the remaining two colors appearing at most once with parts that are odd multiples of 3 . Let $T$ denote the set of partitions into nine distinct colors, with the red and blue parts appearing at most once in multiples of 6 , the green and pink colors appearing at most once with parts congruent to \pm 1 modulo 6 , the orange color appearing at most once with parts congruent to \pm 2 modulo 6 , and the remaining four colors appearing at most once with parts that are odd multiples of 3. Let $D_{S}(N)$ denote the number of partitions of $N$ into distinct elements of $S$, and let $D_{T}(N)$ denote the number of partitions of $N$ into distinct elements of $T$. Then, for all $N \geq 1$,

$$
D_{S}(N)=\frac{1}{2} D_{T}(N)
$$


Proof. Consider once again the parameterizations given in (5.2) and (5.3). By simple elementary algebra, we can check that

$$
\begin{aligned}
& (1-\sqrt{1-\alpha})(1+\sqrt{1-\beta}) \\
= & \frac{-m^{4}+4 m^{3}+18 m^{2}-12 m-9}{16 m^{2}}+\frac{(m+3)(m-1) \sqrt{(m+1)(3-m)}}{4 m \sqrt{m}} \\
= & \alpha^{1 / 4} \beta^{1 / 4}+\{\alpha \beta(1-\alpha)(1-\beta)\}^{1 / 4}+2 \alpha^{1 / 4} \beta^{1 / 4}\{(1-\alpha)(1-\beta)\}^{1 / 8} .
\end{aligned}
$$

Extract the square root on both sides of the resulting identity to obtain the equation

$$
(1-\sqrt{1-\alpha})^{1 / 2}(1+\sqrt{1-\beta})^{1 / 2}=\alpha^{1 / 8} \beta^{1 / 8}+\{\alpha \beta(1-\alpha)(1-\beta)\}^{1 / 8} .
$$

Dividing both sides of $(5.15)$ by $\{\alpha \beta(1-\alpha)(1-\beta)\}^{1 / 8}$ and rearranging terms, we arrive at

$$
\begin{aligned}
& \frac{(1-\beta)^{1 / 12}\left(\beta / q^{3}\right)^{-1 / 6} z_{3}^{1 / 2}(1+\sqrt{1-\beta})^{1 / 2}}{(1-\alpha)^{1 / 12}(\alpha / q)^{-1 / 24}(1-\beta)^{1 / 12}\left(\beta / q^{3}\right)^{-1 / 24}} \\
& \times \frac{1}{z_{3}^{1 / 2}(1-\beta)^{1 / 8}(1-\alpha)^{1 / 24}(1+\sqrt{1-\alpha})^{1 / 6}(1-\sqrt{1-\alpha})^{-1 / 3} q^{1 / 3}} \\
= & \frac{\{\alpha(1-\alpha) / q\}^{-1 / 12}\left\{\beta(1-\beta) / q^{3}\right\}^{-1 / 12}}{(1-\alpha)^{1 / 24}(\alpha / q)^{-1 / 12}(1-\beta)^{1 / 24}\left(\beta / q^{3}\right)^{-1 / 12}}+1,
\end{aligned}
$$

which is equivalent to

$$
\frac{2 \chi^{2}\left(-q^{6}\right) \varphi\left(q^{6}\right)}{\chi(-q) \chi\left(-q^{3}\right) \varphi\left(-q^{6}\right) \chi^{2}\left(-q^{4}\right)}=\frac{\chi^{2}(q) \chi^{2}\left(q^{3}\right)}{\chi\left(-q^{2}\right) \chi\left(-q^{6}\right)}+1,
$$

by (2.10), (2.11), (2.12), (4.2), (4.5), and (4.10). Applying (4.15), we arrive at

$$
\frac{2 \chi^{2}\left(q^{6}\right)}{\chi(-q) \chi\left(-q^{3}\right) \chi^{2}\left(-q^{4}\right)}=\frac{\chi^{2}(q) \chi^{2}\left(q^{3}\right)}{\chi\left(-q^{2}\right) \chi\left(-q^{6}\right)}+1 .
$$

Employing the definition of $\chi$ from (2.3), we can derive a reformulation of the last equation into $q$-products, namely,

$$
\begin{aligned}
& 2(-q ; q)_{\infty}\left(-q^{3} ; q^{3}\right)_{\infty}\left(-q^{4} ; q^{4}\right)_{\infty}^{2}\left(-q^{6} ; q^{12}\right)_{\infty}^{2} \\
= & \left(-q ; q^{2}\right)_{\infty}^{2}\left(-q^{2} ; q^{2}\right)_{\infty}\left(-q^{3} ; q^{6}\right)_{\infty}^{2}\left(-q^{6} ; q^{6}\right)_{\infty}+1 .
\end{aligned}
$$

Equating the coefficients of $q^{N}$ on both sides of the last equation, we finish the proof.

Remark. Theorem 5.9 is equivalent to Conjecture 3.50 in Sandon and Zanello's paper [16].

Example 5.10. Let $N=4$ in Theorem 5.9. Then $D_{S}(4)=5$ and $D_{T}(4)=$ $1+4 \cdot 2+1=10$. The partitions that we seek are given by

$$
\begin{aligned}
& 4_{y}=4_{1}=4_{2}=3_{3}+1_{o}=34+1_{o} ; \\
& 4_{o}=3_{1}+1_{g}=3_{1}+1_{p}=\cdots=3_{4}+1_{p}=\cdots=2_{o}+1_{g}+1_{p} .
\end{aligned}
$$


Theorem 5.11. Let $S$ denote the set of partitions into ten distinct colors, with the red, blue, green, and pink parts appearing at most once in multiples of 6 , the orange color appearing at most once with parts congruent to \pm 1 modulo 6 , the yellow color appearing at most once with parts congruent to \pm 2 modulo 6 , another two colors appearing at most once with parts congruent to \pm 4 modulo 12 , and the remaining two colors appearing at most once with parts that are odd multiples of 3. Let $T$ denote the set of partitions into eleven distinct colors, with the red, blue, green, and pink parts appearing at most once in multiples of 6 , the orange color appearing at most once with parts congruent to \pm 1 modulo 6 , the yellow color appearing at most once with parts congruent to \pm 4 modulo 12 , another two colors appearing at most once with parts that are odd multiples of 3 , and the remaining three colors appearing at most once with parts congruent to \pm 2 modulo 12. Let $D_{S}(N)$ denote the number of partitions of $N$ into distinct elements of $S$, and let $D_{T}(N)$ denote the number of partitions of $N$ into distinct elements of $T$. Then, for all $N \geq 1$,

$$
D_{S}(N)=D_{T}(N-1) \text {. }
$$

Proof. Referring to the formulas (5.14) and (5.15), we can check that

$$
\begin{aligned}
& (1-\sqrt{1-\alpha})^{1 / 2}(1+\sqrt{1-\beta})^{1 / 2} \\
= & (1+\sqrt{1-\alpha})^{1 / 2}(1-\sqrt{1-\beta})^{1 / 2}+2\{\alpha \beta(1-\alpha)(1-\beta)\}^{1 / 8},
\end{aligned}
$$

where $\beta$ has degree 3 over $\alpha$. Next, divide both sides of (5.16) by $2\{\alpha \beta(1-$ $\alpha)(1-\beta)\}^{1 / 8}$ and rearrange terms to obtain the equation

$$
\begin{aligned}
& \frac{(1-\beta)^{1 / 12}\left(\beta / q^{3}\right)^{-1 / 6} z_{3}^{1 / 2}(1+\sqrt{1-\beta})^{1 / 2}}{2(1-\alpha)^{1 / 12}(\alpha / q)^{-1 / 24}(1-\beta)^{1 / 12}\left(\beta / q^{3}\right)^{-1 / 24}} \\
& \times \frac{1}{(1-\alpha)^{1 / 24}(1+\sqrt{1-\alpha})^{1 / 6}(1-\sqrt{1-\alpha})^{-1 / 3} q^{1 / 3} z_{3}^{1 / 2}(1-\beta)^{1 / 8}} \\
= & q \frac{(1-\alpha)^{1 / 12}(\alpha / q)^{-1 / 6} z_{1}^{1 / 2}(1+\sqrt{1-\alpha})^{1 / 2}}{2(1-\alpha)^{1 / 12}(\alpha / q)^{-1 / 24}(1-\beta)^{1 / 12}\left(\beta / q^{3}\right)^{-1 / 24}} \\
& \times \frac{1}{(1-\beta)^{1 / 24}(1+\sqrt{1-\beta})^{1 / 6}(1-\sqrt{1-\beta})^{-1 / 3} q z_{1}^{1 / 2}(1-\alpha)^{1 / 8}}+1 .
\end{aligned}
$$

Utilizing (2.10)-(2.12), (4.2), (4.5), and (4.10), we arrive at

$$
\frac{\chi^{2}\left(-q^{6}\right) \varphi\left(q^{6}\right)}{\chi(-q) \chi\left(-q^{3}\right) \chi^{2}\left(-q^{4}\right) \varphi\left(-q^{6}\right)}=q \frac{\chi^{2}\left(-q^{2}\right) \varphi\left(q^{2}\right)}{\chi(-q) \chi\left(-q^{3}\right) \chi^{2}\left(-q^{12}\right) \varphi\left(-q^{2}\right)}+1,
$$

which can be transformed into

$$
\frac{\chi^{2}\left(q^{6}\right)}{\chi(-q) \chi\left(-q^{3}\right) \chi^{2}\left(-q^{4}\right)}=q \frac{\chi^{2}\left(q^{2}\right)}{\chi(-q) \chi\left(-q^{3}\right) \chi^{2}\left(-q^{12}\right)}+1
$$

by (4.15). Applying the definition of $\chi$ from (2.3), we can convert the last identity into $q$-products, namely,

$$
(-q ; q)_{\infty}\left(-q^{3} ; q^{3}\right)_{\infty}\left(-q^{4} ; q^{4}\right)_{\infty}^{2}\left(-q^{6} ; q^{12}\right)_{\infty}^{2}
$$




$$
=q(-q ; q)_{\infty}\left(-q^{3} ; q^{3}\right)_{\infty}\left(-q^{2} ; q^{4}\right)_{\infty}^{2}\left(-q^{12} ; q^{12}\right)_{\infty}^{2}+1
$$

Equating the coefficients of $q^{N}$ on both sides of the last equation, we finish the proof.

Remark. Theorem 5.11 is equivalent to Conjecture 3.48 in Sandon and Zanello's paper [16].

Example 5.12. Let $N=4$ in Theorem 5.11. Then $D_{S}(4)=5$ and $D_{T}(3)=5$. The illustrative partitions are then given by

$$
\begin{aligned}
& 4_{y}=4_{1}=4_{2}=3_{3}+1_{o}=3_{4}+1_{o} ; \\
& 3_{1}=3_{2}=2_{3}+1_{o}=2_{4}+1_{o}=2_{5}+1_{o} .
\end{aligned}
$$

Theorem 5.13. Let $S$ denote the set of partitions into eight distinct colors, with the red and blue parts appearing at most once with even parts, the green and pink parts appearing at most once with parts congruent to \pm 2 modulo 12 , and the remaining four colors in parts appearing at most once with odd multiples of 3. Let $T$ denote the set of partitions into eight distinct colors, with the red parts appearing at most once with odd parts, the blue and green parts appearing at most once with even parts, the orange parts appearing at most once with odd multiples of 3 , and the remaining four colors appearing at most once with parts in multiples of 12 . If $D_{S}(N)$ denotes the number of partitions of $N$ into distinct elements of $S$, and if $D_{T}(N)$ denotes the number of partitions of $N$ into distinct elements of $T$, then, for all $N \geq 2$,

$$
D_{S}(N)=4 D_{T}(N-2) \text {. }
$$

Proof. Return to (5.2) and (5.3), and use simple elementary algebra to deduce that

$$
-\alpha^{1 / 8}(1-\alpha)^{1 / 8}=-\alpha^{1 / 8}(1-\beta)^{3 / 8}+\beta^{3 / 8}(1-\alpha)^{1 / 8} .
$$

Next, multiply both sides of $(5.17)$ by $\beta^{1 / 8}(1-\beta)^{1 / 8}$ and add $\alpha^{1 / 8} \beta^{1 / 8}$ on both sides of the resulting equation to obtain the equation

$$
\begin{aligned}
& \alpha^{1 / 8} \beta^{1 / 8}-\{\alpha \beta(1-\alpha)(1-\beta)\}^{1 / 8} \\
= & \alpha^{1 / 8} \beta^{1 / 8}-(\alpha \beta)^{1 / 8}(1-\beta)^{1 / 2}+\beta^{1 / 2}(1-\alpha)^{1 / 8}(1-\beta)^{1 / 8} .
\end{aligned}
$$

Using (5.14) on the left-hand side of the last identity, we arrive at

$(1+\sqrt{1-\alpha})^{1 / 2}(1-\sqrt{1-\beta})^{1 / 2}=\alpha^{1 / 8} \beta^{1 / 8}(1-\sqrt{1-\beta})+\beta^{1 / 2}(1-\alpha)^{1 / 8}(1-\beta)^{1 / 8}$.

Divide both sides of $(5.18)$ by $\beta^{1 / 2}(1-\alpha)^{1 / 8}(1-\beta)^{1 / 8}$ to deduce that

$$
\begin{aligned}
& \frac{(1+\sqrt{1-\alpha})^{1 / 2}}{(1-\alpha)^{1 / 8}(1-\beta)^{1 / 8}(1+\sqrt{1-\beta})^{1 / 2}} \\
= & \frac{\alpha^{1 / 8}(1-\sqrt{1-\beta})^{2 / 3}}{(1-\alpha)^{1 / 8}(1-\beta)^{1 / 8}(1+\sqrt{1-\beta})^{1 / 3} \beta^{1 / 24}}+1 .
\end{aligned}
$$


Rearranging terms in the last identity, we find that

$$
\begin{aligned}
& \frac{\sqrt{z_{1}}(1+\sqrt{1-\alpha})^{1 / 2} \sqrt{z_{3}}(1-\beta)^{1 / 8}\left\{\beta(1-\beta) / q^{3}\right\}^{-1 / 6}}{\sqrt{z_{1}}(1-\alpha)^{1 / 8} \sqrt{z_{3}}(1+\sqrt{1-\beta})^{1 / 2}(1-\beta)^{1 / 12}\left(\beta / q^{3}\right)^{-1 / 6}} \\
= & q^{2} \frac{\{\alpha(1-\alpha) / q\}^{-1 / 24}\left\{\beta(1-\beta) / q^{3}\right\}^{-1 / 24}}{(1-\alpha)^{1 / 12}(\alpha / q)^{-1 / 6}(1-\beta)^{1 / 12}(1+\sqrt{1-\beta})^{1 / 3}(1-\sqrt{1-\beta})^{-2 / 3} q^{2}}+1 .
\end{aligned}
$$

Hence, from $(2.10),(2.12),(4.2),(4.5)$, and (4.10), it suffices to prove that

$$
\frac{\varphi\left(q^{2}\right) \varphi\left(-q^{6}\right) \chi^{4}\left(q^{3}\right)}{\varphi\left(-q^{2}\right) \varphi\left(q^{6}\right) \chi^{2}\left(-q^{6}\right)}=4 q^{2} \frac{\chi(q) \chi\left(q^{3}\right)}{\chi^{2}\left(-q^{2}\right) \chi^{4}\left(-q^{12}\right)}+1
$$

which can be transformed into

$$
\frac{\chi^{2}\left(q^{2}\right) \chi^{4}\left(q^{3}\right)}{\chi^{2}\left(-q^{2}\right) \chi^{2}\left(q^{6}\right)}=4 q^{2} \frac{\chi(q) \chi\left(q^{3}\right)}{\chi^{2}\left(-q^{2}\right) \chi^{4}\left(-q^{12}\right)}+1,
$$

by (4.15). Employing above the definition of $\chi$ from (2.3) and Euler's identity (2.13), we can find that

$$
\begin{aligned}
& \frac{\left(-q^{2} ; q^{2}\right)_{\infty}^{2}\left(-q^{2} ; q^{4}\right)_{\infty}^{2}\left(-q^{3} ; q^{6}\right)_{\infty}^{4}}{\left(-q^{6} ; q^{12}\right)_{\infty}^{2}} \\
= & 4 q^{2}\left(-q ; q^{2}\right)_{\infty}\left(-q^{2} ; q^{2}\right)_{\infty}^{2}\left(-q^{3} ; q^{6}\right)_{\infty}\left(-q^{12} ; q^{12}\right)_{\infty}^{4}+1 .
\end{aligned}
$$

Equate the coefficients of $q^{N}$ on both sides of the last equation to finish the proof.

Remark. Theorem 5.13 is equivalent to Conjecture 3.41 in Sandon and Zanello's paper [16].

Example 5.14. Let $N=5$ in Theorem 5.13. Then $D_{S}(5)=4 \cdot 4=16$ and $D_{T}(3)=4$. The partitions that we need are given by

$$
\begin{aligned}
& 3_{1}+2_{r}=\cdots=3_{4}+2_{r}=3_{1}+2_{g}=\cdots ; \\
& 3_{r}=3_{o}=2_{b}+1_{r}=2_{g}+1_{r} .
\end{aligned}
$$

Theorem 5.15. Let $S$ denote the set of partitions into four distinct colors, with the red, blue, green, and orange parts appearing at most once with parts either congruent to \pm 1 modulo 6 or congruent to \pm 4 modulo 12 . Let $T$ denote the set of partitions into four distinct colors, with the red, blue, green, and orange parts appearing at most once with parts either congruent to \pm 1 modulo 6 or congruent to \pm 2 modulo 12 . Let $D_{S}(N)$ denote the number of partitions of $N$ into an odd number of distinct elements of $S$, and let $D_{T}(N)$ denote the number of partitions of $N$ into an odd number of distinct elements of $T$. Then, for all $N \geq 3$,

$$
D_{S}(N)=D_{T}(N-2) \text {. }
$$

Proof. Recall the parameterizations for $\alpha$ and $\beta$ of degree 3 given in (5.2) and (5.3). By simple elementary algebra, we can check that

$$
(1-\sqrt{1-\alpha})(1+\sqrt{1-\beta})-(1+\sqrt{1-\alpha})(1-\sqrt{1-\beta})
$$




$$
\begin{aligned}
& =\frac{(m-1)(3+m) \sqrt{(m+1)(3-m)}}{2 m \sqrt{m}} \\
& =m^{2} \alpha \frac{(1-\alpha)^{3 / 4}}{(1-\beta)^{3 / 4}}-\beta \frac{(1-\alpha)^{3 / 4}}{(1-\beta)^{3 / 4}}+8 \alpha^{1 / 2} \beta^{1 / 2} \frac{(1-\alpha)^{1 / 4}}{(1-\beta)^{1 / 4}} .
\end{aligned}
$$

Next, multiply both sides of the last identity by $q \frac{(1-\beta)^{1 / 4}}{(1-\alpha)^{1 / 4}(\alpha \beta)^{1 / 2}}$, apply the identity $1-\sqrt{1-\alpha}=\frac{\alpha}{1+\sqrt{1-\alpha}}$, and rearrange the resulting identity to obtain the equation

$$
\begin{aligned}
& \frac{q(1-\beta)^{1 / 4} \alpha^{1 / 2}(1+\sqrt{1-\beta})}{(1-\alpha)^{1 / 4} \beta^{1 / 2}(1+\sqrt{1-\alpha})}-m^{2} q \frac{\alpha^{1 / 2}(1-\alpha)^{1 / 2}}{\beta^{1 / 2}(1-\beta)^{1 / 2}} \\
= & \frac{q(1-\beta)^{1 / 4} \alpha^{1 / 6}(1+\sqrt{1-\alpha})^{1 / 3}(1-\sqrt{1-\beta})^{2 / 3}}{(1-\alpha)^{1 / 4} \beta^{1 / 6}(1-\sqrt{1-\alpha})^{2 / 3}(1+\sqrt{1-\beta})^{1 / 3}}-\frac{q(1-\alpha)^{1 / 2} \beta^{1 / 2}}{(1-\beta)^{1 / 2} \alpha^{1 / 2}}+8 q,
\end{aligned}
$$

which can be rewritten as

$$
\begin{aligned}
& \frac{(1-\beta)^{1 / 3}\left(\beta / q^{3}\right)^{-1 / 6}(1-\beta)^{1 / 6}\left(\beta / q^{3}\right)^{-1 / 3} z_{3}(1+\sqrt{1-\beta}) z_{1}(1-\alpha)^{1 / 4}}{(1-\alpha)^{1 / 3}(\alpha / q)^{-1 / 6}(1-\alpha)^{1 / 6}(\alpha / q)^{-1 / 3} z_{1}(1+\sqrt{1-\alpha}) z_{3}(1-\beta)^{1 / 4}} \\
& -\frac{z_{1}^{2}(1-\alpha)^{2 / 3}(\alpha / q)^{1 / 6}(1-\beta)^{1 / 6}\left(\beta / q^{3}\right)^{-1 / 3}}{z_{3}^{2}(1-\beta)^{2 / 3}\left(\beta / q^{3}\right)^{1 / 6}(1-\alpha)^{1 / 6}(\alpha / q)^{-1 / 3}} \\
= & q^{2} \frac{(1-\beta)^{1 / 3}\left(\beta / q^{3}\right)^{-1 / 6}(1-\alpha)^{1 / 12}(1+\sqrt{1-\alpha})^{1 / 3}(1-\sqrt{1-\alpha})^{-2 / 3} q^{2 / 3}}{(1-\alpha)^{1 / 3}(\alpha / q)^{-1 / 6}(1-\beta)^{1 / 12}(1+\sqrt{1-\beta})^{1 / 3}(1-\sqrt{1-\beta})^{-2 / 3} q^{2}} \\
& -q^{2} \frac{z_{1}^{2}(1-\alpha)^{2 / 3}(\alpha / q)^{1 / 6} z_{3}^{2}(1-\beta)^{1 / 6}\left(\beta / q^{3}\right)^{2 / 3}}{z_{3}^{2}(1-\beta)^{2 / 3}\left(\beta / q^{3}\right)^{1 / 6} z_{1}^{2}(1-\alpha)^{1 / 6}(\alpha / q)^{2 / 3}}+8 q .
\end{aligned}
$$

Hence, from (2.8), (2.11), (2.12), (4.2), (4.5), (4.8), and (4.10), it suffices to prove that

$$
\begin{aligned}
& \frac{\chi^{4}\left(-q^{3}\right) \chi^{4}\left(-q^{6}\right) \varphi^{2}\left(q^{6}\right) \varphi^{2}\left(-q^{2}\right)}{\chi^{4}(-q) \chi^{4}\left(-q^{2}\right) \varphi^{2}\left(-q^{6}\right) \varphi^{2}\left(q^{2}\right)}-\frac{f^{4}(-q) \chi^{4}\left(-q^{6}\right)}{f^{4}\left(-q^{3}\right) \chi^{4}\left(-q^{2}\right)} \\
= & q^{2}\left\{\frac{\chi^{4}\left(-q^{3}\right) \chi^{4}\left(-q^{4}\right)}{\chi^{4}(-q) \chi^{4}\left(-q^{12}\right)}-\frac{f^{4}(-q) f^{4}\left(-q^{12}\right)}{f^{4}\left(-q^{3}\right) f^{4}\left(-q^{4}\right)}\right\}+8 q .
\end{aligned}
$$

Using (4.15), we arrive at

$$
\begin{aligned}
& \frac{\chi^{4}\left(-q^{3}\right) \chi^{4}\left(q^{6}\right)}{\chi^{4}(-q) \chi^{4}\left(q^{2}\right)}-\frac{f^{4}(-q) \chi^{4}\left(-q^{6}\right)}{f^{4}\left(-q^{3}\right) \chi^{4}\left(-q^{2}\right)} \\
= & q^{2}\left\{\frac{\chi^{4}\left(-q^{3}\right) \chi^{4}\left(-q^{4}\right)}{\chi^{4}(-q) \chi^{4}\left(-q^{12}\right)}-\frac{f^{4}(-q) f^{4}\left(-q^{12}\right)}{f^{4}\left(-q^{3}\right) f^{4}\left(-q^{4}\right)}\right\}+8 q .
\end{aligned}
$$

Applying the definitions of $f$ and $\chi$ from (2.2) and (2.3), respectively, we can convert the last equation into $q$-products, namely,

$$
\frac{(-q ; q)_{\infty}^{4}\left(-q^{6} ; q^{12}\right)_{\infty}^{4}}{\left(-q^{3} ; q^{3}\right)_{\infty}^{4}\left(-q^{2} ; q^{4}\right)_{\infty}^{4}}-\frac{(q ; q)_{\infty}^{4}\left(q^{6} ; q^{12}\right)_{\infty}^{4}}{\left(q^{3} ; q^{3}\right)_{\infty}^{4}\left(q^{2} ; q^{4}\right)_{\infty}^{4}}
$$




$$
=q^{2}\left\{\frac{(-q ; q)_{\infty}^{4}\left(-q^{12} ; q^{12}\right)_{\infty}^{4}}{\left(-q^{3} ; q^{3}\right)_{\infty}^{4}\left(-q^{4} ; q^{4}\right)_{\infty}^{4}}-\frac{(q ; q)_{\infty}^{4}\left(q^{12} ; q^{12}\right)_{\infty}^{4}}{\left(q^{3} ; q^{3}\right)_{\infty}^{4}\left(q^{4} ; q^{4}\right)_{\infty}^{4}}\right\}+8 q
$$

Equating the coefficients of $q^{N}$ on both sides of the last equation, we finish the proof.

Remark. Theorem 5.15 is equivalent to Conjecture 3.29 in Sandon and Zanello's paper [16].

Example 5.16. Let $N=5$ in Theorem 5.15. Then $D_{S}(5)=D_{T}(3)=4$. The partitions that we want are given by

$$
\begin{aligned}
& 5_{r}=5_{b}=5_{g}=5_{o} \\
& 1_{r}+1_{b}+1_{g}=1_{b}+1_{g}+1_{o}=1_{g}+1_{o}+1_{r}=1_{r}+1_{b}+1_{o} .
\end{aligned}
$$

Theorem 5.17. Let $S$ denote the set of partitions into four distinct colors, with the red and blue parts appearing at most once with parts not congruent to 0 or \pm 2 modulo 12 , the green parts appearing at most once with parts congruent to \pm 2 modulo 12 , and the orange parts appearing at most once with parts congruent to \pm 1 modulo 6 . Let $T$ denote the set of partitions into four distinct colors, with the red and blue parts appearing at most once with parts not congruent to 6 or \pm 4 modulo 12, the green parts appearing at most once with parts congruent to \pm 4 modulo 12, and the orange parts appearing at most once with parts congruent to \pm 1 modulo 6 . Let $D_{S}(N)$ denote the number of partitions of $N$ into an odd number of distinct elements of $S$, and let $D_{T}(N)$ denote the number of partitions of $N$ into distinct elements of $T$. Then, for all $N \geq 2$,

$$
D_{S}(N)=D_{T}(N-2) \text {. }
$$

Proof. Recall the parameterizations for $\alpha$ and $\beta$ of degree 3 given in (5.2) and (5.3). By simple elementary algebra and the equation (5.12), we can find that

$$
(1+\sqrt{1-\alpha})^{1 / 4}(1+\sqrt{1-\beta})^{1 / 4}+(1-\sqrt{1-\alpha})^{1 / 4}(1-\sqrt{1-\beta})^{1 / 4}=2^{1 / 2}\left\{\frac{(1-\beta)^{3}}{1-\alpha}\right\}^{1 / 16} .
$$

Next, applying (5.13) and (5.19), we can also check that

$$
\begin{aligned}
& (1-\sqrt{1-\alpha})^{1 / 4}(1-\sqrt{1-\beta})^{1 / 4}-(1+\sqrt{1-\alpha})^{1 / 4}(1+\sqrt{1-\beta})^{1 / 4} \\
& +\sqrt{1-\beta}\left\{(1+\sqrt{1-\alpha})^{1 / 4}(1+\sqrt{1-\beta})^{1 / 4}+(1-\sqrt{1-\alpha})^{1 / 4}(1-\sqrt{1-\beta})^{1 / 4}\right\} \\
= & m 2^{1 / 2} \frac{(1-\alpha)^{9 / 16}(\alpha \beta)^{1 / 4}}{(1-\beta)^{3 / 16}}+3 \cdot 2^{1 / 2} \frac{\beta^{3 / 8}(1-\alpha)^{3 / 16}(\alpha \beta)^{1 / 4}}{\alpha^{1 / 8}(1-\beta)^{1 / 16}} .
\end{aligned}
$$

Multiplying both sides of $(5.20)$ by $\frac{2^{1 / 2} q \alpha^{1 / 8}(1-\beta)^{1 / 16}}{\beta^{3 / 8}(1-\alpha)^{3 / 16}(\alpha \beta)^{1 / 4}}$ and rearranging the resulting identity, we obtain

$$
2^{1 / 2} q \frac{1+\sqrt{1-\beta}}{(1+\sqrt{1-\alpha})^{1 / 4}(1+\sqrt{1-\beta})^{1 / 4}} \frac{\alpha^{1 / 8}(1-\beta)^{1 / 16}}{\beta^{3 / 8}(1-\alpha)^{3 / 16}}-m 2 q \frac{\alpha^{1 / 8}(1-\alpha)^{3 / 8}}{\beta^{3 / 8}(1-\beta)^{1 / 8}}
$$




$$
=2^{1 / 2} q \frac{1-\sqrt{1-\beta}}{(1-\sqrt{1-\alpha})^{1 / 4}(1-\sqrt{1-\beta})^{1 / 4}} \frac{\alpha^{1 / 8}(1-\beta)^{1 / 16}}{\beta^{3 / 8}(1-\alpha)^{3 / 16}}+6 q,
$$

which is equivalent to

$$
\begin{aligned}
& \frac{2^{1 / 2}\{\alpha(1-\alpha) / q\}^{-1 / 8}(1-\beta)^{1 / 24}\left(\beta / q^{3}\right)^{-1 / 12} z_{3}^{1 / 4}(1+\sqrt{1-\beta})^{1 / 4} z_{1}^{1 / 4}(1-\alpha)^{1 / 16}}{(1-\alpha)^{1 / 8}(\alpha / q)^{-1 / 4}\left\{\beta(1-\beta) / q^{3}\right\}^{-1 / 24} z_{1}^{1 / 4}(1+\sqrt{1-\alpha})^{1 / 4} z_{3}^{1 / 4}(1-\beta)^{1 / 16}} \\
& \times(1-\beta)^{1 / 24}(1+\sqrt{1-\beta})^{1 / 6}(1-\sqrt{1-\beta})^{-1 / 3} q \\
& -\frac{z_{1} 2^{-2 / 3}\{\alpha(1-\alpha) / q\}^{1 / 6} 2^{1 / 2}(1-\alpha)^{1 / 4}(\alpha / q)^{-1 / 8} 2^{1 / 3}(1-\beta)^{1 / 24}\left(\beta / q^{3}\right)^{-1 / 12}}{z_{3} 2^{-4 / 3}(1-\beta)^{1 / 12}\left(\beta / q^{3}\right)^{1 / 3} 2^{1 / 3}(1-\alpha)^{1 / 24}(\alpha / q)^{-1 / 12} 2^{1 / 6}(1-\beta)^{1 / 12}\left(\beta / q^{3}\right)^{-1 / 24}} \\
= & 2 q^{2} \frac{2^{1 / 2}\{\alpha(1-\alpha) / q\}^{-1 / 8} z_{1}^{1 / 4} 2^{-1 / 4}(1+\sqrt{1-\alpha})^{1 / 4} z_{3}^{1 / 2}(1-\beta)^{1 / 8}}{2^{5 / 12}(1-\beta)^{1 / 48}(1+\sqrt{1-\beta})^{1 / 12}(1-\sqrt{1-\beta})^{-1 / 6} q^{1 / 2} 2^{1 / 6}\left\{\beta(1-\beta) / q^{3}\right\}^{-1 / 24}} \\
& \times \frac{1}{2^{2 / 3}(1-\beta)^{1 / 12}\left(\beta / q^{3}\right)^{-1 / 6} z_{1}^{1 / 4}(1-\alpha)^{1 / 16} z_{3}^{1 / 2} 2^{-1 / 2}(1+\sqrt{1-\beta})^{1 / 2}}+6 q .
\end{aligned}
$$

Hence, from (2.9), (2.10), (2.11), (2.12), (4.2), (4.5), (4.8), and (4.10), it suffices to prove that

$$
\begin{aligned}
& \frac{\chi^{3}(q) \chi\left(-q^{6}\right) \sqrt{\varphi\left(q^{6}\right)} \sqrt{\varphi\left(-q^{2}\right)} \chi^{2}\left(-q^{12}\right)}{\chi^{3}\left(-q^{2}\right) \chi\left(q^{3}\right) \sqrt{\varphi\left(-q^{6}\right)} \sqrt{\varphi\left(q^{2}\right)}}-\frac{f^{2}\left(-q^{2}\right) \chi^{3}(-q) \chi\left(-q^{6}\right)}{f^{2}\left(-q^{12}\right) \chi\left(-q^{2}\right) \chi\left(-q^{3}\right)} \\
= & 2 q^{2} \frac{\chi^{3}(q) \sqrt{\varphi\left(q^{2}\right)} \varphi\left(-q^{6}\right)}{\chi\left(q^{3}\right) \chi^{2}\left(-q^{6}\right) \sqrt{\varphi\left(-q^{2}\right)} \varphi\left(q^{6}\right) \chi\left(-q^{12}\right)}+6 q .
\end{aligned}
$$

Using the formula (4.15), we find that

$$
\begin{aligned}
& \frac{\chi^{3}(q) \chi\left(q^{6}\right) \chi^{2}\left(-q^{12}\right)}{\chi^{2}\left(-q^{2}\right) \chi\left(q^{2}\right) \chi\left(q^{3}\right)}-\frac{f^{2}\left(-q^{2}\right) \chi^{3}(-q) \chi\left(-q^{6}\right)}{f^{2}\left(-q^{12}\right) \chi\left(-q^{2}\right) \chi\left(-q^{3}\right)} \\
= & 2 q^{2} \frac{\chi^{3}(q) \chi\left(q^{2}\right)}{\chi\left(q^{3}\right) \chi\left(-q^{2}\right) \chi^{2}\left(q^{6}\right) \chi\left(-q^{12}\right)}+6 q .
\end{aligned}
$$

Applying the definitions of $f$ and $\chi$ from (2.2) and (2.3), we can rewrite the last equation in the form

$$
\begin{aligned}
& \frac{\left(-q^{2} ; q^{2}\right)_{\infty}^{2}\left(-q ; q^{2}\right)_{\infty}^{3}\left(-q^{6} ; q^{12}\right)_{\infty}}{\left(-q^{12} ; q^{12}\right)_{\infty}^{2}\left(-q^{2} ; q^{4}\right)_{\infty}\left(-q^{3} ; q^{6}\right)_{\infty}}-\frac{\left(q^{2} ; q^{2}\right)_{\infty}^{2}\left(q ; q^{2}\right)_{\infty}^{3}\left(q^{6} ; q^{12}\right)_{\infty}}{\left(q^{12} ; q^{12}\right)_{\infty}^{2}\left(q^{2} ; q^{4}\right)_{\infty}\left(q^{3} ; q^{6}\right)_{\infty}} \\
= & 2 q^{2} \frac{\left(-q ; q^{2}\right)_{\infty}^{3}\left(-q^{2} ; q^{2}\right)_{\infty}\left(-q^{2} ; q^{4}\right)_{\infty}\left(-q^{12} ; q^{12}\right)_{\infty}}{\left(-q^{3} ; q^{6}\right)_{\infty}\left(-q^{6} ; q^{12}\right)_{\infty}^{2}}+6 q .
\end{aligned}
$$

Equate the coefficients of $q^{N}$ on both sides of the last equation to complete the proof.

Remark. Theorem 5.17 is equivalent to Conjecture 3.43 in Sandon and Zanello's paper [16]. 
Example 5.18. Let $N=5$ in Theorem 5.17. Then $D_{S}(5)=3+2 \cdot\left(\begin{array}{l}3 \\ 2\end{array}\right)=9$ and $D_{T}(3)=2+2 \cdot 3+1=9$. The partitions that we want are given by

$$
\begin{aligned}
& 5_{r}=5_{b}=5_{o}=3_{r}+1_{r}+1_{g}=\cdots ; \\
& 3_{r}=3_{b}=2_{r}+1_{o}=2_{r}+1_{b}=\cdots=1_{r}+1_{b}+1_{o} .
\end{aligned}
$$

Theorem 5.19. Let $S$ denote the set of partitions into four distinct colors, with the red and blue parts appearing at most once with parts not congruent to 0 or \pm 2 modulo 12, the green parts appearing at most once with parts congruent to \pm 2 modulo 12, and the orange parts appearing at most once with parts congruent to \pm 1 modulo 6 . Let $T$ denote the set of partitions into seven distinct colors, with the red parts appearing at most once with parts that are not odd multiples of 6 , the blue parts appearing at most once with parts in multiples of 3 but not odd multiples of 6 , the green and orange parts appearing at most once with parts congruent to \pm 2 modulo 12 , and the remaining three colors appearing at most once with parts congruent to \pm 4 modulo 12 . Let $D_{S}(N)$ denote the number of partitions of $N$ into an odd number of distinct elements of $S$, and let $D_{T}(N)$ denote the number of partitions of $N$ into distinct elements of $T$. Then, for all $N \geq 2$,

$$
D_{S}(N)=D_{T}(N-1)
$$

Proof. Recall the parameterizations for $\alpha$ and $\beta$ of degree 3 given in (5.2) and (5.3). By simple elementary algebra, (5.13), and (5.19), we know that

$$
(1+\sqrt{1-\alpha})^{1 / 4}(1+\sqrt{1-\beta})^{1 / 4}=\frac{1}{2^{1 / 2}}\left\{\left\{\frac{(1-\beta)^{3}}{1-\alpha}\right\}^{1 / 16}+\left\{\frac{(1-\alpha)^{3}}{1-\beta}\right\}^{1 / 16}\right\} .
$$

Next, we can also check that

$$
\begin{aligned}
& 1-(\alpha \beta)^{1 / 4}+(1-\beta)^{1 / 2}=\frac{(m+1)(3-m)}{4 m}+\frac{(m+1) \sqrt{(m+1)(3-m)}}{4 \sqrt{m}} \\
= & \left\{m 2^{1 / 2}\left\{\frac{(1-\alpha)^{3}}{1-\beta}\right\}^{3 / 16}+2^{3 / 2}\left(\frac{\beta^{3}}{\alpha}\right)^{1 / 8}\left\{\frac{(1-\alpha)^{3}}{1-\beta}\right\}^{1 / 16}\right\}(1+\sqrt{1-\alpha})^{1 / 4}(1+\sqrt{1-\beta})^{1 / 4} .
\end{aligned}
$$

Dividing both sides of $(5.21)$ by $(1+\sqrt{1-\alpha})^{1 / 4}(1+\sqrt{1-\beta})^{1 / 4}$ and rearranging the resulting identity, we obtain

$$
\begin{aligned}
& \frac{(1+\sqrt{1-\beta})^{3 / 4}}{(1+\sqrt{1-\alpha})^{1 / 4}}-m 2^{1 / 2}\left\{\frac{(1-\alpha)^{3}}{1-\beta}\right\}^{3 / 16} \\
= & \frac{(\alpha \beta)^{1 / 4}}{(1+\sqrt{1-\alpha})^{1 / 4}(1+\sqrt{1-\beta})^{1 / 4}}+2^{3 / 2}\left(\frac{\beta^{3}}{\alpha}\right)^{1 / 8}\left\{\frac{(1-\alpha)^{3}}{1-\beta}\right\}^{1 / 16} .
\end{aligned}
$$

Divide both sides of the last identity by $2^{-1 / 2} q^{-1}\left(\frac{\beta^{3}}{\alpha}\right)^{1 / 8}\left\{\frac{(1-\alpha)^{3}}{1-\beta}\right\}^{1 / 16}$ to find that

$$
\frac{2^{1 / 2}\{\alpha(1-\alpha) / q\}^{-1 / 8}(1-\beta)^{1 / 24}\left(\beta / q^{3}\right)^{-1 / 12} z_{3}^{1 / 4}(1+\sqrt{1-\beta})^{1 / 4} z_{1}^{1 / 4}(1-\alpha)^{1 / 16}}{(1-\alpha)^{1 / 8}(\alpha / q)^{-1 / 4}\left\{\beta(1-\beta) / q^{3}\right\}^{-1 / 24} z_{1}^{1 / 4}(1+\sqrt{1-\alpha})^{1 / 4} z_{3}^{1 / 4}(1-\beta)^{1 / 16}}
$$




$$
\begin{aligned}
& \times(1-\beta)^{1 / 24}(1+\sqrt{1-\beta})^{1 / 6}(1-\sqrt{1-\beta})^{-1 / 3} q \\
& -\frac{z_{1} 2^{-2 / 3}\{\alpha(1-\alpha) / q\}^{1 / 6} 2^{1 / 2}(1-\alpha)^{1 / 4}(\alpha / q)^{-1 / 8} 2^{1 / 3}(1-\beta)^{1 / 24}\left(\beta / q^{3}\right)^{-1 / 12}}{z_{3} 2^{-4 / 3}(1-\beta)^{1 / 12}\left(\beta / q^{3}\right)^{1 / 3} 2^{1 / 3}(1-\alpha)^{1 / 24}(\alpha / q)^{-1 / 12} 2^{1 / 6}(1-\beta)^{1 / 12}\left(\beta / q^{3}\right)^{-1 / 24}} \\
= & \frac{2 q\left\{2^{1 / 3}(1-\beta)^{1 / 24}\left(\beta / q^{3}\right)^{-1 / 12}\right\}^{3} 2^{1 / 6}\left\{\beta(1-\beta) / q^{3}\right\}^{-1 / 24}}{2^{1 / 6}(1-\alpha)^{1 / 12}(\alpha / q)^{-1 / 24} 2^{5 / 12}(1-\alpha)^{1 / 48}(1+\sqrt{1-\alpha})^{1 / 12}(1-\sqrt{1-\alpha})^{-1 / 6} q^{1 / 6}} \\
& \times \frac{1}{\left\{2^{1 / 3}(1-\alpha)^{1 / 24}(\alpha / q)^{-1 / 12}\right\}^{2} 2^{5 / 12}(1-\beta)^{1 / 48}(1+\sqrt{1-\beta})^{1 / 12}(1-\sqrt{1-\beta})^{-1 / 6} q^{1 / 2}}+4 q .
\end{aligned}
$$

Hence, from (2.9), (2.10), (2.11), (2.12), (4.2), (4.5), (4.8), and (4.10), it suffices to prove that

$$
\begin{aligned}
& \frac{\chi^{3}(q) \chi\left(-q^{6}\right) \sqrt{\varphi\left(q^{6}\right)} \sqrt{\varphi\left(-q^{2}\right)} \chi^{2}\left(-q^{12}\right)}{\chi^{3}\left(-q^{2}\right) \chi\left(q^{3}\right) \sqrt{\varphi\left(-q^{6}\right)} \sqrt{\varphi\left(q^{2}\right)}}-\frac{f^{2}\left(-q^{2}\right) \chi^{3}(-q) \chi\left(-q^{6}\right)}{f^{2}\left(-q^{12}\right) \chi\left(-q^{2}\right) \chi\left(-q^{3}\right)} \\
= & \frac{2 q \chi^{3}\left(-q^{6}\right) \chi\left(q^{3}\right)}{\chi(-q) \chi^{2}\left(-q^{2}\right) \chi\left(-q^{4}\right) \chi\left(-q^{12}\right)}+4 q .
\end{aligned}
$$

Using (4.15), we find that

$$
\begin{aligned}
& \frac{\chi^{3}(q) \chi\left(q^{6}\right) \chi^{2}\left(-q^{12}\right)}{\chi^{2}\left(-q^{2}\right) \chi\left(q^{2}\right) \chi\left(q^{3}\right)}-\frac{f^{2}\left(-q^{2}\right) \chi^{3}(-q) \chi\left(-q^{6}\right)}{f^{2}\left(-q^{12}\right) \chi\left(-q^{2}\right) \chi\left(-q^{3}\right)} \\
= & \frac{2 q \chi^{3}\left(-q^{6}\right) \chi\left(q^{3}\right)}{\chi(-q) \chi^{2}\left(-q^{2}\right) \chi\left(-q^{4}\right) \chi\left(-q^{12}\right)}+4 q .
\end{aligned}
$$

Applying the definitions of $f$ and $\chi$ from (2.2) and (2.3), we can rewrite the last equation in terms of $q$-products, namely,

$$
\begin{aligned}
& \frac{\left(-q^{2} ; q^{2}\right)_{\infty}^{2}\left(-q ; q^{2}\right)_{\infty}^{3}\left(-q^{6} ; q^{12}\right)_{\infty}}{\left(-q^{12} ; q^{12}\right)_{\infty}^{2}\left(-q^{2} ; q^{4}\right)_{\infty}\left(-q^{3} ; q^{6}\right)_{\infty}}-\frac{\left(q^{2} ; q^{2}\right)_{\infty}^{2}\left(q ; q^{2}\right)_{\infty}^{3}\left(q^{6} ; q^{12}\right)_{\infty}}{\left(q^{12} ; q^{12}\right)_{\infty}^{2}\left(q^{2} ; q^{4}\right)_{\infty}\left(q^{3} ; q^{6}\right)_{\infty}} \\
= & 2 q \frac{(-q ; q)_{\infty}\left(-q^{2} ; q^{2}\right)_{\infty}^{2}\left(-q^{4} ; q^{4}\right)_{\infty}\left(-q^{3} ; q^{6}\right)_{\infty}\left(-q^{12} ; q^{12}\right)_{\infty}}{\left(-q^{6} ; q^{6}\right)_{\infty}^{3}}+4 q .
\end{aligned}
$$

Equate the coefficients of $q^{N}$ on both sides of the last equation to finish the proof.

Remark. Theorem 5.19 is equivalent to Conjecture 3.45 in Sandon and Zanello's paper [16].

Example 5.20. Let $N=5$ in Theorem 5.19. Then $D_{S}(5)=3+2 \cdot\left(\begin{array}{l}3 \\ 2\end{array}\right)=9$ and $D_{T}(4)=4+2+\left(\begin{array}{l}3 \\ 2\end{array}\right)=9$. The partitions relevant to us are given by

$$
\begin{aligned}
& 5_{r}=5_{b}=5_{o}=3_{r}+1_{r}+1_{g}=\cdots ; \\
& 4_{r}=4_{1}=4_{2}=4_{3}=3_{r}+1_{r}=3_{b}+1_{r}=2_{r}+2_{o}=\cdots .
\end{aligned}
$$

Theorem 5.21. Let $S$ denote the set of partitions into seven distinct colors, with the red parts appearing at most once but not in odd multiples of 6 , the blue parts appearing at most once with multiples of 3 , but not odd multiples of 6 , the green and pink colors appearing at most once with parts congruent to \pm 2 modulo 12 , and the remaining three colors appearing at most once with 
parts congruent to \pm 4 modulo 12 . Let $T$ denote the set of partitions into four distinct colors, with the red and blue parts appearing at most once with parts not congruent to 6 or \pm 4 modulo 12, the green color appearing at most once with parts congruent to \pm 4 modulo 12 , and the pink color appearing at most once with parts congruent to \pm 1 modulo 6 . Let $D_{S}(N)$ denote the number of partitions of $N$ into distinct elements of $S$, and let $D_{T}(N)$ denote the number of partitions of $N$ into distinct elements of $T$. Then, for all $N \geq 1$,

$$
D_{S}(N)=D_{T}(N-1) \text {. }
$$

Proof. Applying Theorems 5.17 and 5.19 directly, we can easily complete the proof.

Remark. Theorem 5.21 is equivalent to Conjecture 3.44 in Sandon and Zanello's paper [16].

Example 5.22. Let $N=4$ in Theorem 5.21. Then $D_{S}(4)=4+2+\left(\begin{array}{l}3 \\ 2\end{array}\right)=9$ and $D_{T}(3)=2+2 \cdot 3+1=9$. The corresponding partitions are given by

$$
\begin{aligned}
& 4_{r}=4_{1}=4_{2}=4_{3}=3_{r}+1_{r}=3_{b}+1_{r}=2_{r}+2_{g}=\cdots ; \\
& 3_{r}=3_{b}=2_{r}+1_{p}=\cdots=1_{r}+1_{b}+1_{p} .
\end{aligned}
$$

Theorem 5.23. Let $S$ denote the set of partitions into four distinct colors, with the red and blue parts appearing at most once with parts not congruent to 2 modulo 4, the green parts appearing at most once with parts congruent to \pm 1 modulo 6 , and the orange parts appearing at most once with parts congruent to \pm 4 modulo 12. Let $T$ denote the set of partitions into four distinct colors, with the red and blue parts appearing at most once with parts that are not multiples of 4 , the green parts appearing at most once with parts congruent to \pm 1 modulo 6 , and the orange parts appearing at most once with parts congruent to \pm 2 modulo 12. Let $D_{S}(N)$ denote the number of partitions of $N$ into distinct elements of $S$, and let $D_{T}(N)$ denote the number of partitions of $N$ into an odd number of distinct elements of $T$. Then, for all $N \geq 1$,

$$
D_{S}(N)=D_{T}(N) \text {. }
$$

Proof. Recall the parameterizations for $\alpha$ and $\beta$ of degree 3 given in (5.2) and (5.3). By simple elementary algebra, we can check that

$$
1-(1-\alpha)^{1 / 4}(1-\beta)^{1 / 4}=(\alpha \beta)^{1 / 4}\left\{\frac{\alpha^{3 / 8}}{\beta^{1 / 8}}-\frac{(1-\alpha)^{3 / 8}}{(1-\beta)^{1 / 8}}\right\} .
$$

Multiply both sides of the equation $(5.22)$ by $2^{1 / 2} \frac{(1-\alpha)^{3 / 16}}{(1-\beta)^{1 / 16}}$ to deduce that

$$
\begin{aligned}
& 2^{1 / 2}\left\{\frac{(1-\alpha)^{3}}{1-\beta}\right\}^{1 / 16}-\sqrt{1-\alpha} 2^{1 / 2}\left\{\frac{(1-\beta)^{3}}{1-\alpha}\right\}^{1 / 16} \\
= & -2^{1 / 2}\left\{\frac{(1-\alpha)^{3}}{1-\beta}\right\}^{3 / 16}(\alpha \beta)^{1 / 4}+2^{1 / 2} \frac{\alpha^{3 / 8}}{\beta^{1 / 8}}\left\{\frac{(1-\alpha)^{3}}{1-\beta}\right\}^{1 / 16}(\alpha \beta)^{1 / 4} .
\end{aligned}
$$


Next, applying (5.13) and (5.19), we can also check that

$$
\begin{aligned}
& (1+\sqrt{1-\alpha})^{1 / 4}(1+\sqrt{1-\beta})^{1 / 4}-(1-\sqrt{1-\alpha})^{1 / 4}(1-\sqrt{1-\beta})^{1 / 4} \\
& -\sqrt{1-\alpha}\left\{(1+\sqrt{1-\alpha})^{1 / 4}(1+\sqrt{1-\beta})^{1 / 4}+(1-\sqrt{1-\alpha})^{1 / 4}(1-\sqrt{1-\beta})^{1 / 4}\right\} \\
= & -2^{1 / 2}\left\{\frac{(1-\alpha)^{3}}{1-\beta}\right\}^{3 / 16}(\alpha \beta)^{1 / 4}+2^{1 / 2} \frac{\alpha^{3 / 8}}{\beta^{1 / 8}}\left\{\frac{(1-\alpha)^{3}}{1-\beta}\right\}^{1 / 16}(\alpha \beta)^{1 / 4} .
\end{aligned}
$$

Multiplying both sides of $(5.23)$ by $\frac{2^{1 / 2} \beta^{1 / 8}(1-\beta)^{1 / 16}}{\alpha^{3 / 8}(1-\alpha)^{3 / 16}(\alpha \beta)^{1 / 4}}$ and rearranging the resulting identity, we find that

$$
\begin{aligned}
& 2^{1 / 2} \frac{1-\sqrt{1-\alpha}}{(1-\sqrt{1-\alpha})^{1 / 4}(1-\sqrt{1-\beta})^{1 / 4}} \frac{\beta^{1 / 8}(1-\beta)^{1 / 16}}{\alpha^{3 / 8}(1-\alpha)^{3 / 16}} \\
= & 2^{1 / 2} \frac{1+\sqrt{1-\alpha}}{(1+\sqrt{1-\alpha})^{1 / 4}(1+\sqrt{1-\beta})^{1 / 4}} \frac{\beta^{1 / 8}(1-\beta)^{1 / 16}}{\alpha^{3 / 8}(1-\alpha)^{3 / 16}}-2 \frac{\beta^{1 / 8}(1-\alpha)^{3 / 8}}{\alpha^{3 / 8}(1-\beta)^{1 / 8}}+2,
\end{aligned}
$$

which is equivalent to

$$
\begin{aligned}
& \frac{2 \cdot 2^{1 / 2}\{\alpha(1-\alpha) / q\}^{-1 / 8} 2^{5 / 12}(1-\beta)^{1 / 48}(1+\sqrt{1-\beta})^{1 / 12}(1-\sqrt{1-\beta})^{-1 / 6} q^{1 / 2}}{2^{5 / 4}(1-\alpha)^{1 / 16}(1+\sqrt{1-\alpha})^{1 / 4}(1-\sqrt{1-\alpha})^{-1 / 2} q^{1 / 2} 2^{1 / 6}\left\{\beta(1-\beta) / q^{3}\right\}^{-1 / 24}} \\
= & \frac{2^{1 / 2}\{\alpha(1-\alpha) / q\}^{-1 / 8} 2(1-\alpha)^{1 / 8}(\alpha / q)^{-1 / 4} z_{1}^{3 / 4} 2^{-3 / 4}(1+\sqrt{1-\alpha})^{3 / 4} z_{3}^{1 / 4}(1-\beta)^{1 / 16}}{2^{1 / 6}\left\{\beta(1-\beta) / q^{3}\right\}^{-1 / 24} 2^{1 / 3}(1-\beta)^{1 / 24}\left(\beta / q^{3}\right)^{-1 / 12} z_{1}^{3 / 4}(1-\alpha)^{3 / 16} z_{3}^{1 / 4} 2^{-1 / 4}(1+\sqrt{1-\beta})^{1 / 4}} \\
& -\frac{2^{1 / 2}(1-\alpha)^{1 / 4}(\alpha / q)^{-1 / 8} 2(1-\alpha)^{1 / 8}(\alpha / q)^{-1 / 4}}{2^{1 / 6}(1-\beta)^{1 / 12}\left(\beta / q^{3}\right)^{-1 / 24} 2^{1 / 3}(1-\beta)^{1 / 24}\left(\beta / q^{3}\right)^{-1 / 12}}+2 .
\end{aligned}
$$

Hence, from (2.10), (2.11), (2.12), (4.2), (4.5), and (4.10), it suffices to prove that

$$
2 \frac{\chi^{3}(q) \chi\left(-q^{12}\right)}{\chi^{3}\left(-q^{4}\right) \chi\left(q^{3}\right)}=\frac{\chi^{3}(q) \chi^{3}\left(-q^{2}\right) \varphi^{1 / 2}\left(-q^{6}\right) \varphi^{3 / 2}\left(q^{2}\right)}{\chi\left(q^{3}\right) \chi\left(-q^{6}\right) \varphi^{1 / 2}\left(q^{6}\right) \varphi^{3 / 2}\left(-q^{2}\right)}-\frac{\chi^{3}(-q) \chi^{3}\left(-q^{2}\right)}{\chi\left(-q^{3}\right) \chi\left(-q^{6}\right)}+2 .
$$

Using the formula (4.15), we find that

$$
2 \frac{\chi^{3}(q) \chi\left(-q^{12}\right)}{\chi^{3}\left(-q^{4}\right) \chi\left(q^{3}\right)}=\frac{\chi^{3}(q) \chi^{3}\left(q^{2}\right)}{\chi\left(q^{3}\right) \chi\left(q^{6}\right)}-\frac{\chi^{3}(-q) \chi^{3}\left(-q^{2}\right)}{\chi\left(-q^{3}\right) \chi\left(-q^{6}\right)}+2 .
$$

Applying the definition of $\chi$ from (2.3), we can rewrite the last equation in the shape

$$
\begin{aligned}
& 2 \frac{\left(-q ; q^{2}\right)_{\infty}^{3}\left(-q^{4} ; q^{4}\right)_{\infty}^{3}}{\left(-q^{12} ; q^{12}\right)_{\infty}\left(-q^{3} ; q^{6}\right)_{\infty}} \\
= & \frac{\left(-q ; q^{2}\right)_{\infty}^{3}\left(-q^{2} ; q^{4}\right)_{\infty}^{3}}{\left(-q^{6} ; q^{12}\right)_{\infty}\left(-q^{3} ; q^{6}\right)_{\infty}}-\frac{\left(q ; q^{2}\right)_{\infty}^{3}\left(q^{2} ; q^{4}\right)_{\infty}^{3}}{\left(q^{6} ; q^{12}\right)_{\infty}\left(q^{3} ; q^{6}\right)_{\infty}}+2 .
\end{aligned}
$$

Equate the coefficients of $q^{N}$ on both sides of the last equation to finish the proof.

Remark. Theorem 5.23 is equivalent to Conjecture 3.47 in Sandon and Zanello's paper [16]. 
Example 5.24. Let $N=5$ in Theorem 5.23. Then $D_{S}(5)=3+3^{2}+2 \cdot\left(\begin{array}{l}3 \\ 2\end{array}\right)=18$ and $D_{T}(5)=3+2 \cdot\left(\begin{array}{l}3 \\ 2\end{array}\right)+\left(\begin{array}{l}3 \\ 2\end{array}\right) \cdot 3=18$. The partitions that we want are given by

$$
\begin{aligned}
& 5_{r}=5_{b}=5_{g}=4_{o}+1_{g}=\cdots=3_{r}+1_{r}+1_{g}=\cdots ; \\
& 5_{r}=5_{b}=5_{g}=3_{r}+1_{r}+1_{g}=\cdots=2_{r}+2_{o}+1_{g}=\cdots .
\end{aligned}
$$

Acknowledgements. The authors are grateful to Ae Ja Yee for initially suggesting this study, to Nayandeep Deka Baruah for useful correspondence, and to Michael Somos for uncovering a couple errors in an earlier draft.

\section{References}

[1] G. E. Andrews, The Theory of Partitions, Encycl. Math. and Its Appl. Vol. 2, AddisonWesley, Reading, 1976; reissued by Cambridge University Press, Cambridge, 1998.

[2] G. E. Andrews and B. C. Berndt, Ramanujan's Lost Notebook. Part I, Springer, New York, 2005.

[3] G. E. Andrews and K. Eriksson, Integer Partitions, Cambridge University Press, Cambridge, 2004.

[4] N. D. Baruah and B. C. Berndt, Partition identities and Ramanujan's modular equations, J. Combin. Theory Ser. A 114 (2007), no. 6, 1024-1045.

[5] _ Partition identities arising from theta function identities, Acta Math. Sin. (Engl. Ser.) 24 (2008), no. 6, 955-970.

[6] N. D. Baruah and B. Boruah, Colored partition identities conjectured by Sandon and Zanello, Ramanujan J., to appear.

[7] B. C. Berndt, Ramanujan's Notebooks. Part III, Springer-Verlag, New York, 1991.

[8] _ Number Theory in the Spirit of Ramanujan, American Mathematical Society, Providence, RI, 2006.

[9] _ Partition-theoretic interpretations of certain modular equations of Schröter, Russell, and Ramanujan, Ann. Comb. 11 (2007), no. 2, 115-125.

[10] B. C. Berndt and R. R. Zhou, Identities for partitions with distinct colors, Ann. Combin., to appear.

[11] W. Chu and L. Di Claudio, Classical Partition Identities and Basic Hypergeometric Series, Quaderno 6/2004 del Dipartimento di Matematica "Ennio De Giorgi", Università degli Studi di Lecce, 2004, Edizioni del Grifo, Lecce.

[12] H. M. Farkas and I. Kra, Partitions and theta constant identities, in The Mathematics of Leon Ehrenpreis, Contemp. Math. No. 251, pp. 197-203, American Mathematical Society, Providence, RI, 2000.

[13] S. Kim, Bijective proofs of partition identities arising from modular equations, J. Combin. Theory Ser. A 116 (2009), no. 3, 699-712.

[14] S. Ramanujan, Notebooks, (2 volumes), Tata Institute of Fundamental Research, Bombay, 1957.

[15] C. Sandon and F. Zanello, Warnaar's bijection and colored partition identities. I, J. Combin. Theory Ser. A 120 (2013), no. 1, 28-38.

[16] - Warnaar's bijection and colored partition identities. II, Ramanujan J. 33 (2014), no. $1,83-120$.

[17] S. O. Warnaar, A generalization of the Farkas and Kra partition theorem for modulus 7, J. Combin. Theory Ser. A 110 (2005), no. 1, 43-52. 
Bruce C. Berndt

Department of Mathematics

UNIVERSITY OF ILLINOIS

1409 West Green Street, Urbana, IL 61801, USA

E-mail address: berndt@illinois.edu

Roberta R. ZHOU

School of Mathematics and Statistics

Northeastern University at Qinhuangdao

Hebei 066004, P. R. China

School of Mathematical Sciences

DALIAN University OF TECHNOLOGY

Dalian 116024, P. R. China

E-mail address: zhourui@neuq.edu.cn 